\title{
Identification of Turbomachinery Noise Sources via Processing Beamforming Data Using Principal Component Analysis
}

\author{
Bence Fenyvesi ${ }^{1 *}$, Csaba Horváth ${ }^{1}$ \\ 1 Department of Fluid Mechanics, Faculty of Mechanical Engineering, Budapest University of Technology and Economics, \\ H-1111 Budapest, Bertalan Lajos street 4-6, Hungary \\ * Corresponding author, e-mail: fenyvesi@ara.bme.hu
}

Received: 15 May 2021, Accepted: 14 September 2021, Published online: 14 December 2021

\begin{abstract}
Complex turbomachinery systems produce a wide range of noise components. The goal is to identify noise source categories, determine their characteristic noise patterns and locations. Researchers can then use this information to quantify the impact of these noise sources, based on which new design guidelines can be proposed. Phased array microphone measurements processed with acoustic beamforming technology provide noise source maps for pre-determined frequency bands (i.e., bins) of the investigated spectrum. However, multiple noise generation mechanisms can be active in any given frequency bin. Therefore, the identification of individual noise sources is difficult and time consuming when using conventional methods, such as manual sorting. This study presents a method for combining beamforming with Principal Component Analysis (PCA) methods in order to identify and separate apart turbomachinery noise sources with strong harmonics. The method is presented through the investigation of Counter-Rotating Open Rotor (CROR) noise sources. It has been found that the proposed semi-automatic method was able to extract even weak noise source patterns that repeat throughout the data set of the beamforming maps. The analysis yields results that are easy to comprehend without special prior knowledge and is an effective tool for identifying and localizing noise sources for the acoustic investigation of various turbomachinery applications.
\end{abstract}

Keywords

principal component analysis, beamforming, noise source pattern, pattern identification

\section{Introduction}

Noise emission has become an important issue in turbomachinery-related applications and research due to the ever-growing demand for improvements in consumer satisfaction, basic comfort, along with increasingly stringent regulatory practices $[1,2]$. As significant noise source reduction can only be accomplished if the generation mechanisms are understood, the development needs to begin with the localization and identification of noise sources.

A state-of-the-art method generally used for the localization of noise sources is acoustic beamforming technology. Frequency domain beamforming results provide information regarding the attributes of various noise source groups as a function of the frequency. Measurement data is obtained by a phased array microphone system. In a phased array microphone system, a set number of microphones are positioned according to a known pattern. From the known microphone locations, the phase and amplitude differences between the recorded signals of the microphones can be obtained. Based on the calculated shifts, the direction of arrival of acoustic wavefronts originating from sound sources in front of the phased array can be estimated [3]. Upon processing the measurement data using beamforming, noise source patterns pertaining to frequency bins of a given frequency range are visualized in the form of beamforming maps. Beamforming maps provide a distribution of the noise sources within a given plane or space. In order to understand the underlying noise generation mechanisms behind the various noise sources, beamforming maps are to be examined both individually and as a series of beamforming maps.

For turbomachinery systems, the interpretation of individual beamforming maps or a sizable series of them is usually a complicated task, as many of the noise source groups are related to the rotation of the rotor (or rotors) in some manner. Consequently, there can often be more than one noise source in any particular frequency bin. 
Moreover, turbomachinery noise sources are often localized to the same general area, creating overlaps at times. Thus, finding a connection between a spatial noise source pattern and its corresponding noise generation mechanism may not always be a straightforward task. Consequently, it can be hard to decide which noise source group to associate a given noise source pattern with [3-5]. The identification of spatial patterns associated with less energetic noise sources can be especially challenging, since their detection is often impeded by more dominant noise generation mechanisms. The extent of the tonal peaks rising above the broadband noise (i.e., the peak-to-noise ratio or peak-to-broadband ratio) can also be relatively small. Consequently, the applicable plot dynamic range on the beamforming maps might be smaller than ideal, potentially resulting in valuable information being lost, which makes it even more challenging to identify the noise source patterns of the various noise source groups.

For sufficient breakdown of an examined frequency range, beamforming is to be used with narrow frequency bands for each bin. However, as narrowband beamforming investigations usually result in a sizable number of frequency bins, the in-depth analysis of a data set can be rather time consuming while requiring extensive experience and knowledge of the noise generation mechanisms under investigation [5]. A noise source group is identified when its characteristic noise source pattern, location, and frequency of appearance are known. Then, the data set of beamforming maps can be simultaneously investigated together with the spectrum created from the peak values of the beamforming maps (referred to as the Beamforming peaks, or BF peaks for short, in this report). The above allows for the separation of noise sources into specific groups, as formerly demonstrated in the literature [4-6].

The above issues regarding the identification of various noise sources are addressed herein. The study applies modal decomposition methods, i.e., Principal Component Analysis (PCA) [7, 8] and Common-base Proper Orthogonal Decomposition (CPOD) [9], in combination with beamforming methods. The goal is to be able to pinpoint the locations and then identify the characteristic noise source patterns associated with specific noise source groups without a time consuming and subjective manual sorting process.

The proposed method is demonstrated herein by investigating the cyclically reoccurring shaft order noise sources of a Counter-Rotating Open Rotor (CROR) aircraft engine, which has two blade sets that rotate in opposite directions, as shown in Fig. 1. Shaft orders, otherwise known

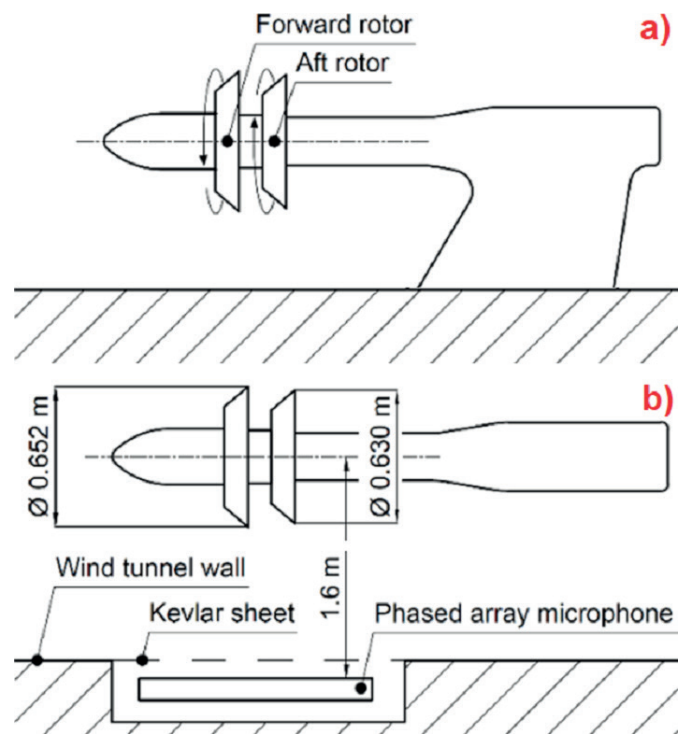

Fig. 1 Schematic drawing of the measurement setup: a) side view (the viewpoint of the array); b) top view

as once-per-rev tones, have been linked in the literature to occurrences repeating once every revolution, or multiples thereof (at higher harmonics of the once-per-rev) [5, 10-12]. These occurrences are usually associated with blade-toblade inconsistencies of a given blade row, e.g., measurement instrumentation mounted on one of the blades, minor blade deformities, etc. [11]. Noise sources associated with the underlying generation mechanism do not tend to be dominant; thus shaft order noise sources are a proper and challenging test subject for the proposed combined identification method. The investigation results will present how capable the proposed method is of detecting the characteristic noise source patterns of these less energetic sources. For the particular CROR test case investigated herein, it is known that measurement instrumentation has been mounted on one of the aft rotor blades and that shaft order tones are localized to the pressure side of the aft rotor near the blade tip [5]. This information will be used in order to verify the results of the proposed identification method.

As a first step of the investigation, beamforming maps have been created from the phased array microphone measurement data set of the chosen CROR test case using a beamforming process appropriate for the given test configuration. For each frequency bin under investigation, a beamforming map is created. These noise source maps are essentially matrices containing the beamforming levels for every grid point of the investigated domain. The beamforming levels provide information regarding noise source amplitudes on the investigated plane. Next, as an alternative to the formerly used manual analysis of the output of 
the beamforming process [5], PCA is applied to the beamforming maps (examined as a function of the frequency herein). The output of the method is that important features of the spatial noise patterns associated with the noise generation mechanisms under investigation are extracted. Hence, the identification of the associated noise sources is made possible. Particular emphasis is placed herein on POD post-processing in the Common-based sense [9], which is an extension of the regular PCA method [7, 8].

An introductory study pertaining to the basic concepts of this process has been presented by Fenyvesi et al. [10], which has been further developed in [13] and then applied in the examination of the blade passing frequency noise in [14]. As the development of the method has been continuous, the various publications have built on the results presented in the earlier reports, implementing further developments, testing, and significant further improvements to the method over time, which are included in the finalized method summarized in this report. It should be noted, that the collective term Proper Orthogonal Decomposition formerly used in the publications to describe the processing procedure has been subsequently changed to Principal Component Analysis. The replacement was made in order to avoid confusion resulting from associations having their roots in the common use of POD in the analyses of velocity fields.

As a result of the proposed method, a significant decrease in subjectivity and an increase in repeatability and automation have been achieved. Therefore, further analysis, grouping, and even ranking of the noise sources is made possible even for a relatively inexperienced analyst.

\section{Principal Component Analysis}

\subsection{Background and analytical description}

PCA (also known as Karhunen-Loève expansion $[15,16]$ and Proper Orthogonal Decomposition [17]) has been applied in numerous scientific fields, such as molecular biology [18], meteorology [19], especially for the recognition and separation of patterns and significant components in a data set related to various phenomena, such as the investigation of complex velocity fields [19-21]. PCA has recently been combined with wavelet-based beamforming methods in CPU cooling fan acoustic imaging for the investigation of a time series of beamforming maps at various frequencies of interest [22].

Generally, PCA determines the characteristic degrees of freedom as contained in the underlying basis, referred to as principal components or the modes of the given problem. As it is an algorithmic method, it decreases the use of subjective judgment, as well as the time needed to analyze a data set. Upon performing PCA, if the raw data basis is comprised of multiple subgroups of information, a common-base POD of the data set allows for a quantitative comparison of these various subgroups [9]. When a data set is processed with PCA and CPOD, information regarding the relative energy contributions of the pre-determined components can be associated with spatial patterns of the original data set.

The matrices that make up the input data set are given in vector form, $\boldsymbol{b}^{i}(i=1 \ldots N)$. Each of the values of these vectors is pertaining to an element of their respective matrices. The vectors are collected over the range of the $N$ input matrices into a collective matrix $\boldsymbol{Q}$, as shown in Eq. (1), which is then further converted into a square covariance matrix $\boldsymbol{R}$ of the data according to Eq. (2). Then, the solution of the corresponding eigenvalue problem (Eq. (3)) provides the matrix $\Psi=\left[\phi_{1} \phi_{2} \ldots \phi_{N}\right]$ of $\phi_{j}$ eigenvectors and the $\lambda_{j}$ eigenvalues, which are arranged in decreasing order, i.e., $\lambda_{j}>\lambda_{j+1}$. The eigenvectors are commonly referred to as PCA modes, which are a set of orthogonal modes. Their respective eigenvalues convey how well each eigenvector captures the original data.

$$
\begin{aligned}
& \boldsymbol{Q}=\left[\begin{array}{lll}
\boldsymbol{b}^{1} \boldsymbol{b}^{2} \ldots \boldsymbol{b}^{\boldsymbol{N}} & \\
\boldsymbol{R} & =\boldsymbol{Q} \boldsymbol{Q}^{T} \\
\boldsymbol{R} \Psi=\lambda \Psi
\end{array}\right.
\end{aligned}
$$

The PCA modes correspond to modal patterns, which are likewise arranged in decreasing order. Consequently, the first few modes are the most important in terms of capturing the energy of the original data set. The $P_{j}$ energy contribution of each mode to the overall power can be determined by dividing the $\lambda_{j}$ eigenvalues by the Euclidean norm of the eigenvalue matrix according to Eq. (4).

$P_{j}=\frac{\lambda_{j}}{\|\lambda\|}$

The contribution of each PCA mode $\phi_{j}$ to the input vectors $\boldsymbol{b}^{i}$ can be determined in terms of weighting coefficients as shown in Eq. (5). Additionally, the individual original matrices can be expressed as a linear combination of the modes and their corresponding weighting coefficients according to Eq. (6). Note that the variance of any mode's coefficient across the $N$ input matrices can also be calculated from the corresponding eigenvalue by dividing 
it by the total number of matrices, as shown in Eq. (7). Recall that the PCA approach does not change the number of degrees of freedom, such that $N$ input matrices lead to $N$ PCA modes.

$$
\begin{aligned}
& \boldsymbol{a}^{i}=\boldsymbol{\Psi} \boldsymbol{b}^{i} \\
& \boldsymbol{b}^{i}=\sum_{j=1}^{N} \boldsymbol{a}_{j}^{i} \phi_{j}=\Psi^{T} \boldsymbol{a}^{i} \\
& \mu_{j}^{N}=\frac{1}{N} \sum_{i=1}^{N}\left(\boldsymbol{a}_{j}^{i}\right)^{2} \underline{\underline{\operatorname{def}}} \frac{\lambda_{j}}{N}
\end{aligned}
$$

\subsection{Expansion to the Common-base processing (CPOD)}

In cases, where the underlying basis of the raw data is comprised of multiple subgroups, the use of the whole data set allows for a quantitative comparison between the subgroups as described in [9]. Via the CPOD-based post-processing of the input data set, the impact of pre-determined matrix-subgroups related to each other can be quantified. Subsequently, characteristic spatial patterns associated with them can also be identified within one particular experiment. Take an experiment, in which two particular subsets, $A$ and $B$ of the original data set have been determined. These two subsets are comprised of the reduced number of input matrices $N^{A}$ and $N^{B} . N^{A}+N^{B}=N$ gives the total number of input matrices. The relative energy contributions of the subsets to the modes is then derived from the sectional variances according to Eqs. (8) and (9), where the superscripts $A$ and $B$ of $\boldsymbol{a}_{j}$ refer to the contributing matrices from the given subsets. In accordance with Eq. (7), the sums of the sectional variances, by definition, add up to the eigenvalues of the respective modes divided by the number of input matrices as shown in Eq. (10).

$$
\begin{aligned}
& \mu_{j}^{N^{A}}=\frac{1}{N^{A}} \sum_{A=1}^{N^{A}}\left(\boldsymbol{a}_{j}^{A}\right)^{2} \\
& \mu_{j}^{N^{B}}=\frac{1}{N^{B}} \sum_{B=1}^{N^{B}}\left(\boldsymbol{a}_{j}^{B}\right)^{2} \\
& \mu_{j}^{N^{A}}+\mu_{j}^{N^{B}}=\mu_{j}^{N} \underline{\underline{\operatorname{def}}} \frac{\lambda_{j}}{N}
\end{aligned}
$$

\section{Measurement setup}

The acoustic measurements processed in this report have been performed on the open rotor propulsion rig in the NASA Glenn Research Center $9 \times 15 \mathrm{ft}$ low-speed wind tunnel $[4,6]$ on a CROR configuration of roughly $1 / 7^{\text {th }}$ scale. The model has been equipped with the F31/
A31 historical baseline blade set, of which further information can be found in [23]. Of the two blade rows, the Forward $(F)$ has 12 blades, having a diameter of $0.652 \mathrm{~m}$, while the $\operatorname{Aft}(A)$ rotor consists of 10 blades with a diameter of $0.630 \mathrm{~m}$. Fig. 1 shows a sketch of the measurement setup, on which the basic metrics of the equipment are also included. The test configuration to be investigated herein is that of an isolated CROR (not having a pylon). It has been examined for the take-off nominal condition, for which regulations with respect to aircraft external noise are becoming more stringent [2]. As for the directions of rotation for the rotors, as seen from the upstream direction, the forward rotor rotates in the clockwise direction, while the aft rotor rotates in the counter-clockwise direction. The rotational speed of the rotors, when corrected for standard day operating conditions, has been 6450 RPM. The Mach number of the flow has been Ma 0.2 , while the angle of attack of the flow with regard to the test rig has been $0^{\circ}$. These measurements have been a part of an extensive research test campaign comprised of aerodynamic and aeroacoustic investigations. Further details regarding the test set-up and the test matrix can be found in [4, 6, 23].

Aeroacoustic measurements have been carried out using an OptiNAV Array48 phased array microphone system [24]. The signals from the 48 flush-mounted microphones have been simultaneously recorded at a sampling rate of $96 \mathrm{kHz}$, and a sampling time of $45 \mathrm{~s}$ has been applied. A camera has been built into the center of the array plate. Upon taking a photo of the field of view of the phased array, the beamforming maps can be superimposed on the image of the measurement setup. In order to remove the microphones from the flow the phased array has been mounted in a cavity along the wind tunnel walls. The microphones have been placed behind a Kevlar ${ }^{\circledR}$ sheet, facing the open rotor test rig. The distance between the center plane of the test rig and the phased array has been $1.6 \mathrm{~m}$.

\section{The input data set}

\subsection{Additional noise source groups}

Other than the shaft order tones previously mentioned in the Introduction, two additional important noise source groups need to be considered when investigating the CROR test case used in this study. The first one is rotating coherent noise sources, which are expected to be the most dominant noise sources of the present CROR test case. Rotating coherent noise sources are associated with distinctive reoccurring frequency values of two kinds: interaction tones and Blade Passing Frequencies (BPF) 
tones $[5,6,25]$. BPF tones are associated with the rotation of the blades (related to the BPF), and shaft order tones are associated with the rotation of the rotors (related to the once-per-rev), overlaps exist between the frequency bins of their harmonics. Therefore, there will be a considerable number of frequency bins in which BPF tones, interaction tones, and shaft order tones can all be present.

Regarding the number of occurrences, a somewhat larger noise source category is that of broadband noise sources. CROR broadband noise is generally related to three main subgroups: leading edge, trailing edge, and blade tip noise [26-28]. Due to their underlying noise generation mechanisms, these noise sources are located on the surface of a rotating element, and are localized to the same position for a relatively wide frequency range. According to the literature, the relative impact of broadband noise on the noise level of a typical CROR configuration increases at higher frequencies, where the amplitudes of tonal sources suffer a stronger decay [29].

\subsection{Beamforming}

In order to carry out the identification of noise sources, the first step is to carry out beamforming and create the noise source maps, which will serve as an input for the PCA based processing described in Section 2. In the current investigation, functional beamforming [30] has been performed. A custom bandwidth of $19.1 \mathrm{~Hz}$ has been utilized, which has divided the frequency range between each BPF of the aft rotor (BPFA) into 50 equal bins. The frequency range investigated herein starts at the $1^{\text {st }}$ BPFA and ends at the $10^{\text {th }}$

As mentioned previously in Section 1, shaft order tones are cyclically reoccurring noise sources and are associated with once-per-rev frequencies. Therefore, the fundamental shaft order frequency of the present CROR configuration (and, by extension, of all turbomachinery configurations) can be calculated according to Eq. (11), which gives a frequency value of $107.5 \mathrm{~Hz}$. Consequently, the fundamental blade passing frequencies are the $12^{\text {th }}$, and the $10^{\text {th }}$ harmonics of this once-per-rev frequency, respectively, as the forward rotor has 12 , while the aft rotor has 10 blades. The center frequencies of the bins in which shaft order tones are expected to appear can be determined using the BPF of the rotor with which they are associated (in our case the aft rotor), and the Blade number of that rotor $\left(B_{A}\right)$. Thus, for the currently investigated CROR test case, a shaft order tone is expected to appear in any $5^{\text {th }}$ frequency bin under investigation. However, as shaft order tones are not the most energetic noise sources of the given test case, their frequency bins can potentially be dominated by noise source patterns pertaining to other noise generation mechanisms.

$f_{S}=\frac{B P F_{A}}{B_{A}}=\frac{\mathrm{RPM}}{60}$

As mentioned previously, for this particular test case, the noise generation of the shaft order tones is a direct result of a measurement instrumentation mounted on one of the aft rotor blades. Consequently, it is to be localized to the pressure side of the aft rotor, which is below the axis on the provided figures.

Fig. 2 presents beamforming maps associated with three different shaft order frequency harmonics of the investigated frequency range. The top of the figure shows the Power Spectral Density (PSD) spectrum created from the BeamForming peak (BFpeak) values, the maximum beamforming values on each beamforming map. In the spectrum, the frequency is given as a function of the fundamental shaft order frequency. Therefore 20 shaft order peaks appear between each major tic of the diagram. It can be seen that many of the shaft order tones can be characterized as having relatively small tonal peaks in the PSD spectrum. Subplots a) to c) of Fig. 2 show the beamforming maps of
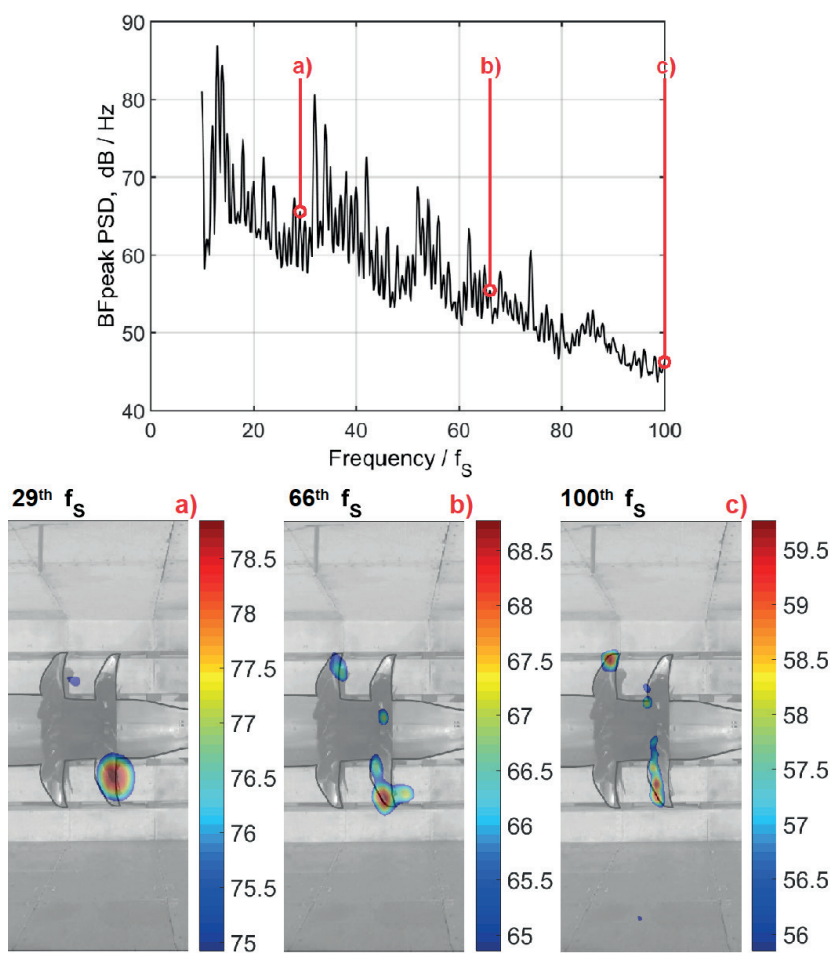

Fig. 2 Beamforming results for shaft order tones with an applied dynamic range of $4 \mathrm{~dB}$ 
the examined frequency bins marked with red circles on the spectrum. The noise source patterns are superimposed over the measurement setup, and the outline of the CROR is highlighted for clarity. For this particular figure, the beamforming maps are plotted using a $4 \mathrm{~dB}$ dynamic range with respect to the beamforming peak values of the maps. Based on the information provided above, the shaft order noise sources associated with the shaft order frequency harmonics presented here are expected to appear below the axis on the aft rotor. By examining the beamforming map shown in subplot a) of Fig. 2, the identification of the shaft order pattern is quite simple, as a single noise source dominates the presented frequency bin and no overlap or spatial interference between multiple noise sources is present. However, for higher harmonics (shown in subplots b) and c)), multiple noise source patterns of elongated shape can be seen, which overlap with the shaft order noise source. At the same time, additional noise source patterns also appear on the forward rotor. Therefore, it can be seen that identifying the noise sources associated with the shaft order tones is rather difficult in certain frequency bins.

In Fig. 3 beamforming maps are shown for the frequency bin of the shaft order harmonic presented in subplot c) of Fig. 2 with multiple dynamic range settings. It can be concluded that choosing the proper dynamic range is highly important for the correct manual identification of a noise source pattern. If its value is too small, valuable information can be lost, while if it is too large, the spatial interference of other noise source patterns and sidelobes can make the task problematic or at times impossible. The proposed beamforming map processing method helps in mitigating the adverse effects associated with the application of various dynamic ranges.

Examining the beamforming maps of the CROR test case, it can be seen that many factors can influence the results and therefore need to be looked at in greater detail. Therefore, an initial investigation has been conducted on
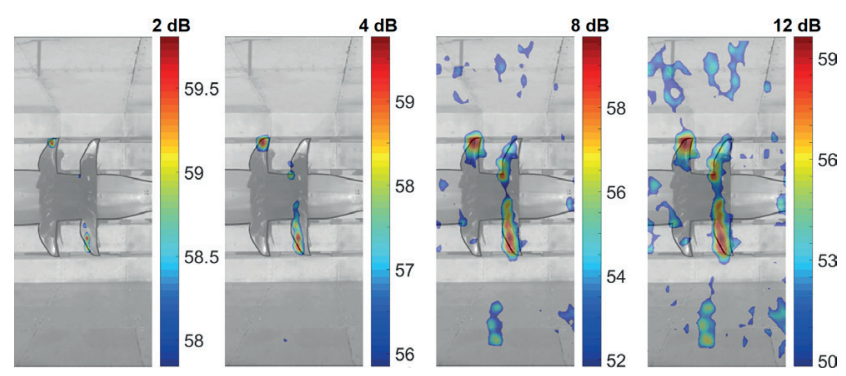

Fig. 3 Beamforming maps of the $100^{\text {th }}$ harmonic of $f_{S}$ with various dynamic range settings an artificially generated data set of a smaller scale. These artificial beamforming maps take after the appearance, features, and properties of the noise sources of the original data set. By analyzing these model test cases, the behavior of maps containing examples of shaft order tones can be assessed with PCA technologies in a controlled environment.

The spectral, as well as the spatial surroundings of CROR shaft order tones need to be examined, in order to generate suitable inputs for the model test case investigations. In Fig. 4 the enlarged spectral surroundings of the shaft order tone pertaining to the $41^{\text {st }}$ harmonic of the $f_{S}$ are presented using a $4 \mathrm{~dB}$ dynamic range. The BFpeak PSD spectrum is marked with a continuous narrow black line, while the frequency bin dominated by a shaft order tone is marked by a vertical dashed red line. Rotating broadband noise sources (marked with a continuous gray line) can have similar noise patterns over several consecutive maps. The cause of this is that the noise sources on the beamforming maps can be localized to the same area on the surface of the aft rotor for a relatively wide frequency range. Depending on the applied dynamic range, these noise sources can be hidden in the spectra, only appearing in frequency bins in which none of the tonal components are significant.
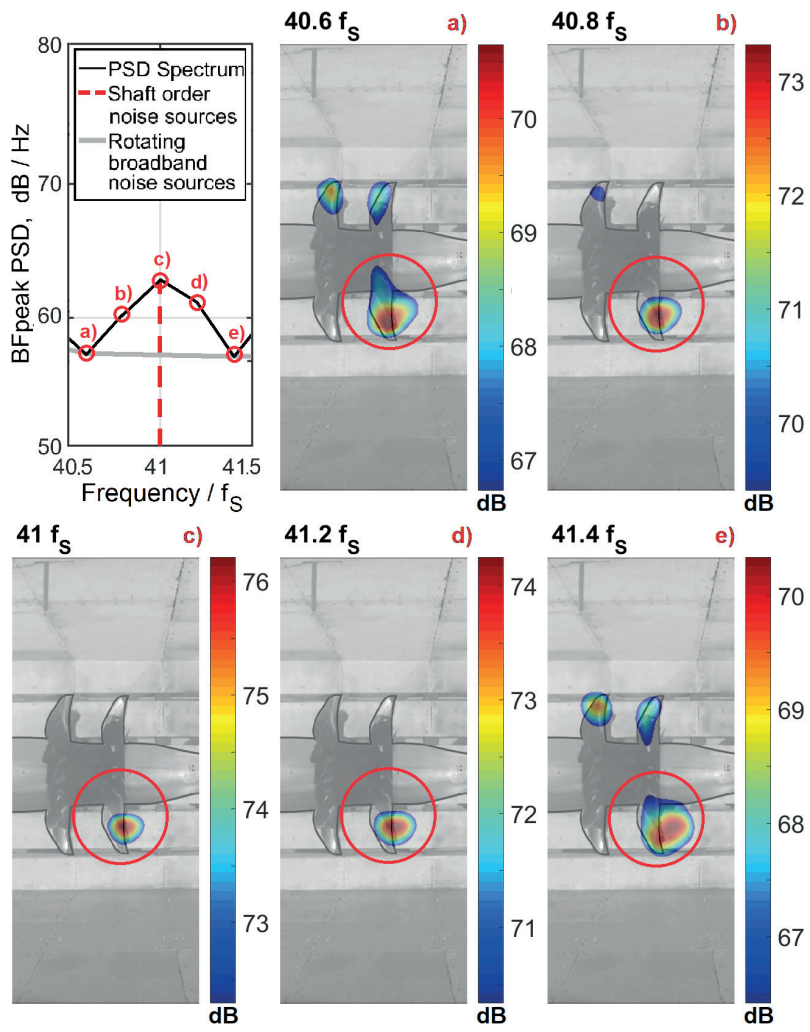

Fig. 4 The enlarged spectral and spatial surroundings of a shaft order tone 
In Fig. 4, data points of two neighboring frequencies on the left-, as well as on the right-hand sides of the shaft order peak are shown together with the peak. These are marked with red circles in the PSD spectrum and letters a)-e), with c) being the peak. It can be observed when investigating these frequency bins on the PSD spectrum, that the tone shows a spectral broadening effect, causing a reduction in the magnitude of the shaft order peak in favor of a distributed spectral hump around the tone frequency. This so-called haystacking has been observed in many acoustic measurements including far-field measurements of turbofan and jet engines [31-33], and is the result of processing records having a finite-duration. The haystacking effect can also be noticed on the beamforming maps of the neighboring frequency bins of the center peak (shown in subplots a) to e) in Fig. 4). The immediate spatial surroundings of the location of the shaft order tone investigated in the present study are marked with red circles on the beamforming maps. It can be seen that for subplots a) and e) of Fig. 4 that the dominant noise sources of these frequency bins are localized to the trailing edge of the forward rotor and to a large portion of the leading edge of the pressure side of the aft rotor, having a somewhat elongated shape as compared to the concentrated noise pattern of the investigated shaft order tone. Therefore, it can be stated, that the noise generation mechanism corresponding to these maps is that of broadband noise sources. Another noise source present in these bins is located above the CROR axis on the leading edge of the aft rotor, which is associated with the interaction of the wake of the forward rotor and the aft rotor. As for subplot c), it displays a noise source that is concentrated to a specific location which can be said to be characteristic of the shaft order tones for the investigated test case. However, due to haystacking, the shaft order noise source also appears on the beamforming maps of subplots b) and d).

The spectral and the spatial characteristics of the shaft order tone of the current CROR test case have been presented above. It can be concluded that in order to identify a noise source pattern properly, a considerable amount of prior knowledge and experience is needed with regard to the following topics: potential noise generation mechanisms, potential locations of appearance of various noise sources, their usual spectral and spatial traits, and the properties of potentially overlapping sources. The identification process is also highly time consuming for a large set of data, and it can be regarded as subjective. The method presented herein provides a means for identifying the characteristic noise source patterns of a desired group of frequencies (and their harmonics) while also eliminating the shortcomings mentioned above.

\subsection{Model test cases}

When investigating complex phenomena, it is common practice to create a simplified representation of the phenomena to carry out studies. Since the present study aims to propose a new means of analysis and processing of beamforming data, it is crucial to investigate the effectiveness and operational properties of the method on data sets of a smaller scale. Therefore, three different model test cases have been created.

When determining the spatial noise and spectral patterns of the maps of the model test cases, the haystacking effect, and the characteristic shapes of CROR and broadband noise sources have been taken into account. The investigation presented in [13] has been further developed herein in order to provide an adequate representation of the phenomena. Five characteristic "beamforming maps" have been created for each of the model test cases investigated here. An example is shown in Fig. 5. The noise source pattern having a bold, elongated "L-shape" aims to represent the broadband component, while the more concentrated circle imitates the characteristics of a shaft order tone. The resulting set of data is then comprised of a repetition of the five characteristic maps seen in Fig. 5, resulting in 60 maps for each of the model test cases as seen in Fig. 6. The intention is to mimic the cyclically reoccurring attributes of the shaft order noise component on the PSD spectra. In Fig. 6 the artificial BFpeak spectra of the three model test cases are shown. Different height ratios of the peaks have been set relative to the broadband level for each of the three different model test cases. The model test case for which the peak-to-broadband ratio is the lowest will be referred to as $L O W$. This particular test case is meant to mimic the spectral and spatial behavior of less energetic shaft order noise sources, which nearly blend into the broadband. On the

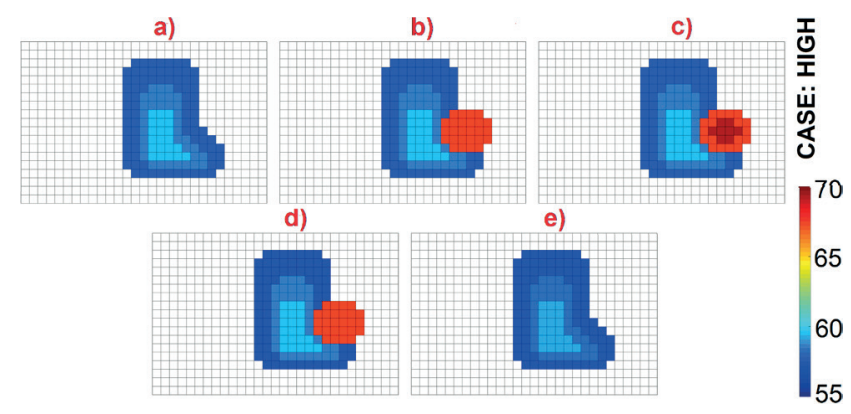

Fig. 5 A sequence of source maps of model test case HIGH 

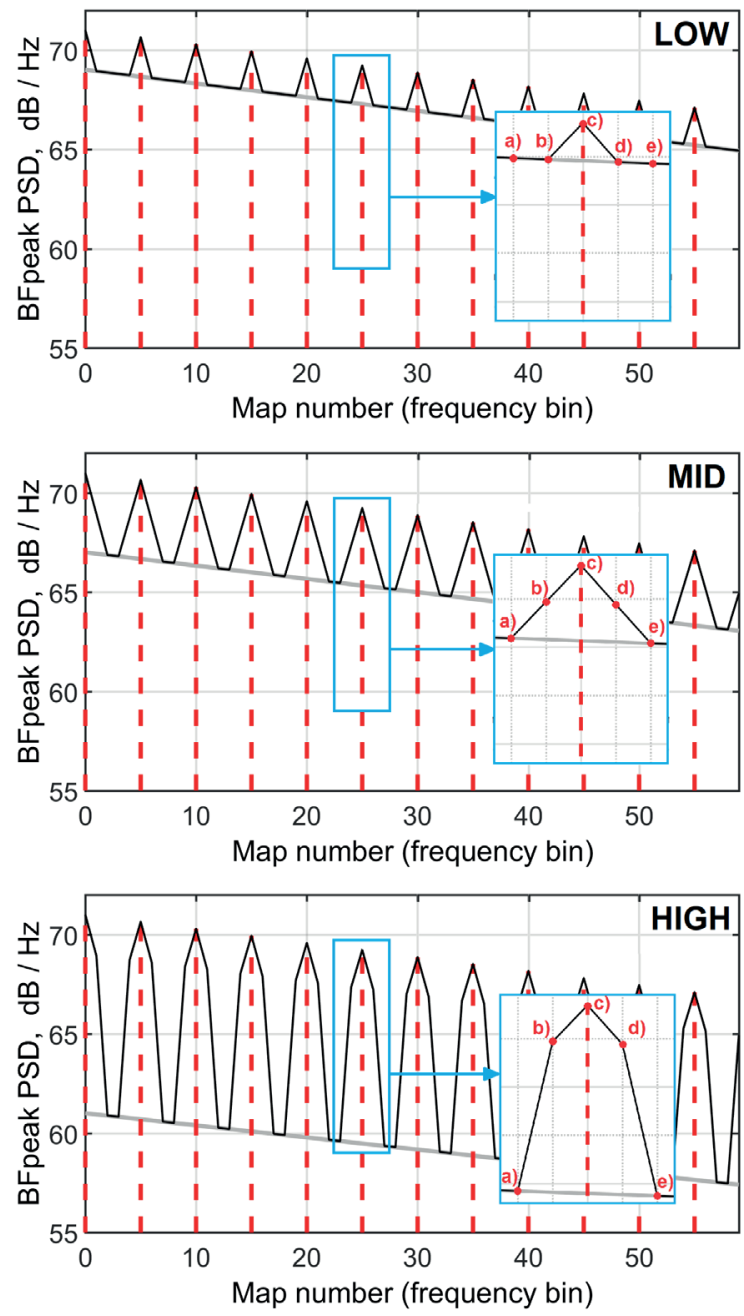

Fig. 6 BFpeak PSD Spectra of the model test cases

contrary, the model test case referred to as $H I G H$ depicts the opposite phenomenon, i.e., tonal shaft order peaks rising high out of the broadband. A data set referred to as MID with the combined attributes of the two previous model test cases has also been created.

The applied dynamic range can also be adjusted later according to the purposes of the investigation of the model test cases. To imitate the gradual amplitude decrease with increasing frequency observed for the CROR test case, an overall amplitude decrease of $0.1 \%$ per map has also been introduced to the values of the maps of the model test cases. Consequently, the present study also goes beyond what has been done previously in [13].

\section{Implementation of the PCA and CPOD approaches}

The current study goes beyond what is seen in the literature (see Section 2), applying a combination of beamforming with PCA and CPOD to recognize and identify reoccurring noise sources. Although the inputs of PCA are usually scalar or vector field snapshots expressed as a function of time, they are in this case beamforming noise source maps presented as a function of frequency in order to find the principal components of the CROR beamforming data set.

According to the description presented in Section 2, the beamforming maps of the frequency bins are given herein in vector form, which will be referred to as the beamforming vectors $\left(\boldsymbol{b}^{i}(i=1 \ldots N)\right)$. The elements of the beamforming vectors are the beamforming values of their respective noise source maps, with each value pertaining to a grid point of the inspected acoustic field. Then, PCA analysis is performed on the data set. In the present context these PCA modes correspond to modal noise patterns, which are arranged in decreasing order according to their importance in terms of capturing the energy of the acoustic source field. Then, the weighting coefficients are calculated (see Eq. (5)) in order to determine the contribution of each of the PCA modes to the input beamforming vectors (and thus the input beamforming maps).

Then the CPOD-based post-processing is applied herein to the data set of the beamforming maps in order to quantify the impact of the frequency bins (and consequently noise source patterns) related to the cyclically reoccurring shaft order tones and subsequently identify their characteristic noise source patterns. The fact that these noise sources appear in a well-defined repetitive pattern throughout the entire frequency range, i.e. shaft orders are associated with the rotational speeds of the rotors (onceper-rev tones and their harmonics), plays a substantial role in the way in which their inclusion in the CPOD analysis of the combined identification process presented in this paper has been implemented. When applying CPOD to identify the shaft order noise patterns of the present CROR test case, two particular subsets of data have been determined and examined. The first subset comprises the beamforming maps of every frequency bin which, originating from their cyclical occurrence, could contain a shaft order tone (even when they are not the most dominant noise sources of their respective frequency bins). These bins can easily be identified using the formula presented in Eq. (11). For the currently investigated CROR test case, every $5^{\text {th }}$ beamforming map falls into this category. The second subset is comprised of the maps of every frequency bin which, strictly looking at it from a spectral perspective, cannot be associated with the characteristic frequencies of shaft order tones. Therefore, the two subsets are comprised of source maps $N^{S}$ and $N^{O}$ where superscript $S$ denotes the 
Shaft order tones, while superscript $O$ refers to the subgroup of all the Other frequency bins $\left(N^{S}+N^{O}=\mathrm{N}\right)$. Therefore, Eqs. (8) to (10) of Subsection 2.2 are also to be modified accordingly.

Furthermore, it is important to be able to identify whether a noise pattern occurs predominantly due to the investigated noise source group. Therefore, apart from the several significant improvements made to the basic ideas of the process presented in [10], the concept of the CPOD maps has also been developed and implemented. In order to identify whether a spatial pattern on the PCA maps occurs predominantly due to shaft order tones (hence subset $S$ ), the actual energy ratio of the sectional variances of the two subsets $\mu_{j}^{N^{S}} / \mu_{j}^{N}$ in a particular mode should be determined. Then the calculated energy ratio can be compared to the value of the expected evenly distributed contribution $N^{S} / N$ of the shaft order subset, which is $1 / 5$ for the present CROR test case.

Consequently, the energy ratio values above or below this value of expected contribution indicate whether the respective patterns are related to shaft order tones or not, thus pinpointing the mode or modes in which the characteristic noise source patterns of shaft order tones appear. The energy ratios can also be used in a subsequent step to visualize the relative impacts of the subsets on the modal noise source patterns as shown in Eqs. (12) and (13). These weighted modal noise patterns are described and will be referred to as CPOD vectors $\boldsymbol{C} j$ herein. The visualizations of the CPOD vectors are referred to herein as CPOD maps. As a summary of the main steps of the described identification process, a flow chart can be seen in Fig. 7.

$$
\begin{gathered}
\boldsymbol{C}_{j}^{S}=\frac{\mu_{j}^{N^{S}}}{\mu_{j}^{N}} \frac{N}{N^{S}} \phi_{j} \\
\boldsymbol{C}_{j}^{O}=\frac{\mu_{j}^{N^{O}}}{\mu_{j}^{N}} \frac{N}{N^{O}} \phi_{j}
\end{gathered}
$$

\section{PCA and CPOD analyses}

\subsection{Model test cases}

PCA and CPOD analyses have been performed on the data sets of the model test cases. As mentioned earlier, as a result of localizing noise sources using beamforming methods noise source maps usually contain sidelobes that are not associated with true noise source locations, therefore are present as undesirable excess information which needs to be suppressed or eliminated in order to be able to investigate the beamforming maps properly. The power

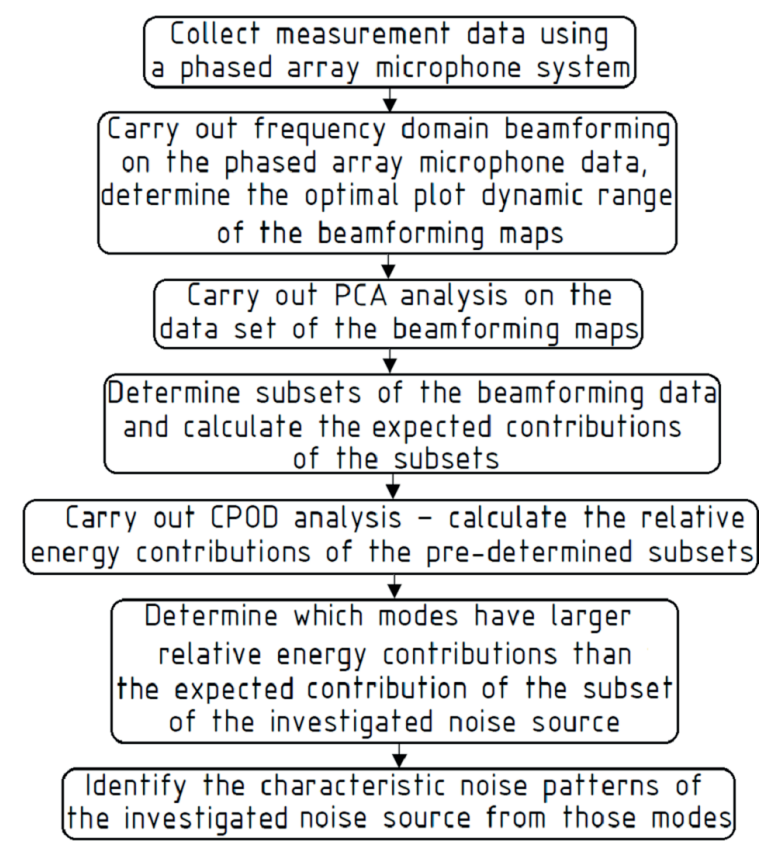

Fig. 7 Flow chart of the identification method

density of the sidelobes is less compared to the mainlobes. Hence the amplitude of the sidelobes is lower than that of the mainlobes, which are associated with the true noise sources of a frequency bin. Various beamforming algorithms, such as the functional beamforming method [30] used herein, can reduce the sidelobe levels to some extent. In addition, a common way of eliminating sidelobes from the beamforming maps is to plot them using a specified dynamic range. The value of the dynamic range is set with respect to the BFpeak (maximum) value of each map. Consequently, if a proper dynamic range is determined for a given test case, irrelevant information can be filtered out while relevant information can be emphasized and shown on the output noise source maps of the beamforming technique. During the PCA and CPOD investigations of the model test cases, the effects of setting various dynamic ranges on the beamforming maps (which serve as an input for the identification method of this study) have been examined. Therefore, values of 2, 4, 8 and $12 \mathrm{~dB}$ have been set with respect to the BFpeak.

The modal noise patterns (PCA and CPOD maps), of the pre-defined subsets $(S$ and $O$ ) created from the beamforming maps of model test case $H I G H$ using a $4 \mathrm{~dB}$ dynamic range are shown in Fig. 8. For this case, it has been found that the first three modes contain the majority of the energy. In the top row, the PCA maps associated with the total energy contained in each mode can be seen, while in the middle and bottom rows of the figure, the CPOD maps are presented, which visualize the 
relative contributions of the subsets to the overall power according to Eqs. (12) and (13). The name of the model test case "HIGH" in the case of Fig. 8 is given on the left hand side together with the applied dynamic range of the input beamforming maps. Information with regard to the relative energy contributions of the modes and about the subsets they consist of is given in Fig. 9 in the form of barplots. The bar-plot on the left hand side displays the relative energy contributions normalized by the total power contained in the underlying basis, while for the plot in the middle, the values are normalized using the energy contained in their respective modes. The relative energy contributions from the bins of the shaft order frequencies are marked with black color, while the contributions of all the other frequency bins are marked with gray.

In order to identify whether a noise pattern on a PCA map occurs predominantly in the frequency bins of the shaft order tones $(S)$ or all the other bins $(O)$, the evenly distributed, hence the expected contribution associated with the shaft order subset has to be determined. A mentioned previously in Subsection 2.2, the peak value of a shaft order tone occurs in a well-defined, repetitive pattern, which in our case is every $5^{\text {th }}$ frequency bin $\left(N^{S} / N=1 / 5\right)$. Therefore, a corresponding relative energy contribution of $20 \%$ indicates an evenly distributed contribution. A solid red line marks this expected contribution in Fig. 9. Sectional variance values above or below this red line indicate whether the respective patterns are shaft-order dominated (i.e., stronger than what is expected) or not. This information can be used to determine the modes that are to be looked at in order to identify the noise source patterns associated
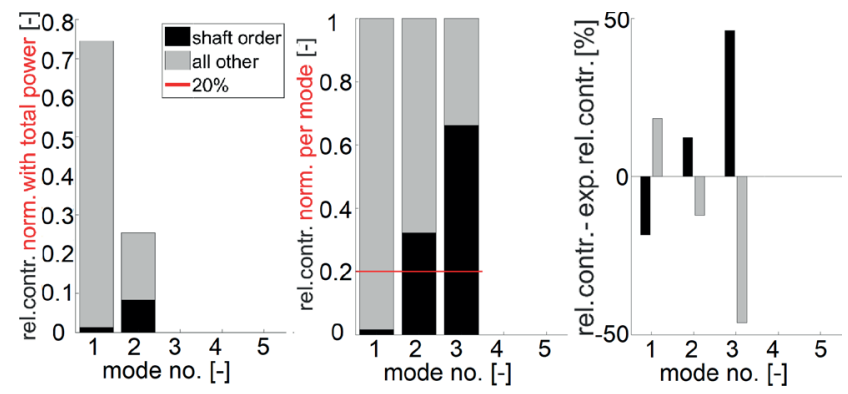

Fig. 9 Relative energy contributions, model test case HIGH, 4 dB

with the currently investigated (i.e., the shaft order) noise sources. In this investigation, the standard PCA maps are also weighted using this relationship, resulting in the CPOD maps. The bar plot on the right-hand side of Fig. 9 displays the percentage difference of the relative energy contributions of the subsets from the values of their evenly distributed contributions. Suppose the difference value associated with the shaft order (black color) subset is positive for a given mode. In that case, it can be stated that the spatial noise patterns of the beamforming maps for shaft order frequency bins have had an effect, which is larger than expected for that mode.

When analyzing the CPOD results, the values to be looked at should first be those pertaining to the relationship of the relative and the expected contributions. It can be seen in Fig. 9, that for the current test case, the energy contribution of the shaft order noise pattern is almost negligible for Mode 1. Thus the absolute (non-dimensional) values on the CPOD map of subset $S$ (shown in the second row of the first column in Fig. 8) are quite small when compared to the CPOD map values of subset $O$. According

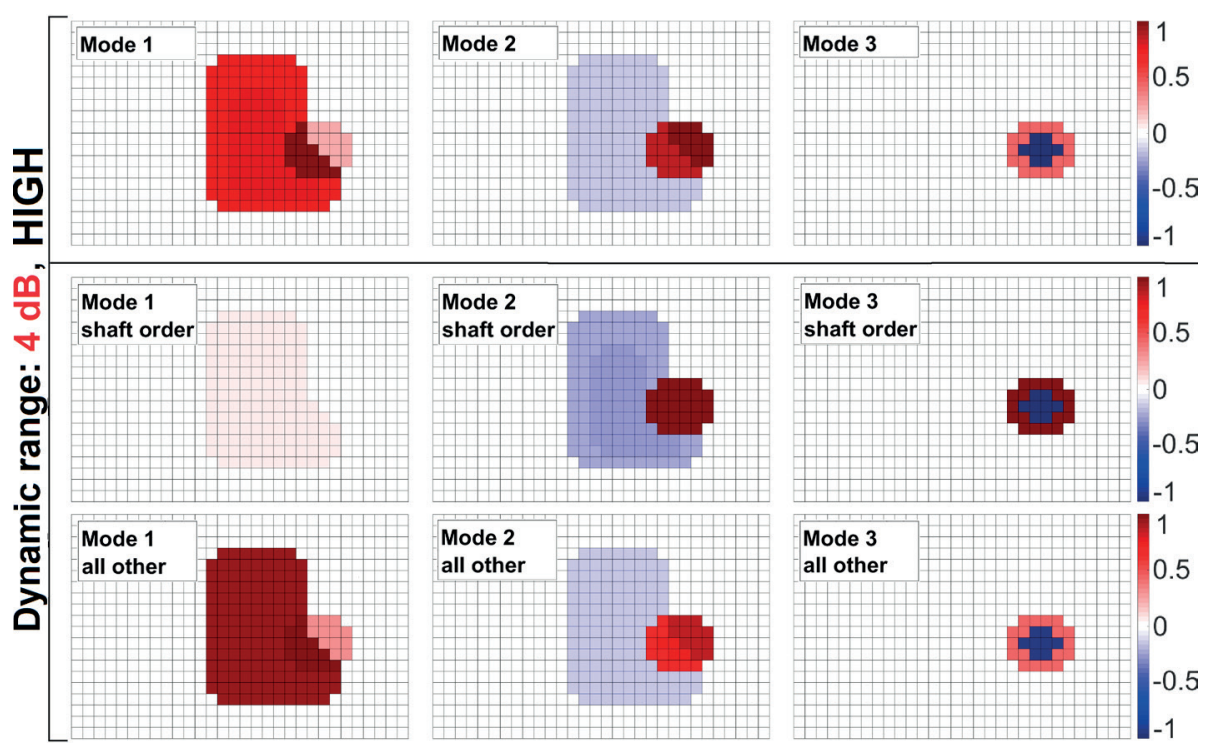

Fig. 8 Modal noise patterns, model test case HIGH, 4 dB 
to these results, it can be stated that the shaft order noise pattern cannot be identified in Mode 1. When examining Modes 2 and 3, it can be seen that the difference values of the relative energy contributions and the expected contributions are positive for both of them. However, the deviation value is approximately four times higher for Mode 3 than for Mode 2. Consequently, the shaft order pattern is expected to appear with fewer additional patterns resulting from non-shaft order sources in Mode 3 than in Mode 2.

Provided that no additional information about the current model test case has been available other than the frequency values of the investigated noise sources, the following can be concluded according to the identification process based on the proposed combined method of this study. That the noise source pattern associated with shaft order tones can be found in Mode 3 and, in part, in Mode 2. As the data sets of the model test cases have been artificially generated, the characteristics of the input beamforming maps are known; the results can be verified by comparing them to the CPOD maps of the modes identified by the CPOD method (i.e., Modes 2 and 3). It can be concluded that the modal noise pattern highlighted in Mode 3 is indeed the shaft order pattern (see Fig. 5), and therefore the identification method has proven to be successful. It can also be seen that the pattern associated with the broadband component is dominant in Mode 1, which is also in agreement with the findings of the analysis, i.e., that subset $O$ (all the other) is the primary contributor of that mode. However, there are certain pattern overlaps on the PCA and CPOD maps, the origins of which need to be explained. In Mode 1, while the elongated, L-shaped noise source pattern pertaining to the broadband component is undoubtedly dominant, the concentrated spot of the shaft order tone is also visible with a lower amplitude. This is due to the haystacking phenomenon, which causes the input beamforming maps of the distinct noise source patterns to be somewhat "contaminated" by one another. Hence an overlap will be present in the various modes. Haystacking is also the reason why the relative energy contributions in Fig. 8 are not made up entirely of only the contribution of one subset. For Mode 2, the overlapping is even more conspicuous. However, separating the patterns is still possible, since they are displayed with opposite signs by the CPOD method. In summary, the identification method is rather straightforward, quick, and simple, while a small number of factors need to be kept in mind during the analysis.

The effect of changing the dynamic range used when creating the input beamforming maps for the three model test cases has also been tested. The results are presented using the CPOD maps of their shaft order subsets (being the noise source under investigation), together with the bar-plots pertaining to the relationships of the expected and the actual relative energy contributions of the subsets (see Figs. 10 to 14).

At first glance it can be stated, that the modal noise patterns and relative energy contributions for each of the modes of the model test cases are the most similar for the ones for which the dynamic range of $12 \mathrm{~dB}$ has been applied (see Figs. 13 and 14). In contrast, the modal noise patterns are all different for the model test cases with the

\section{Dynamic range: $2 \mathrm{~dB}$}

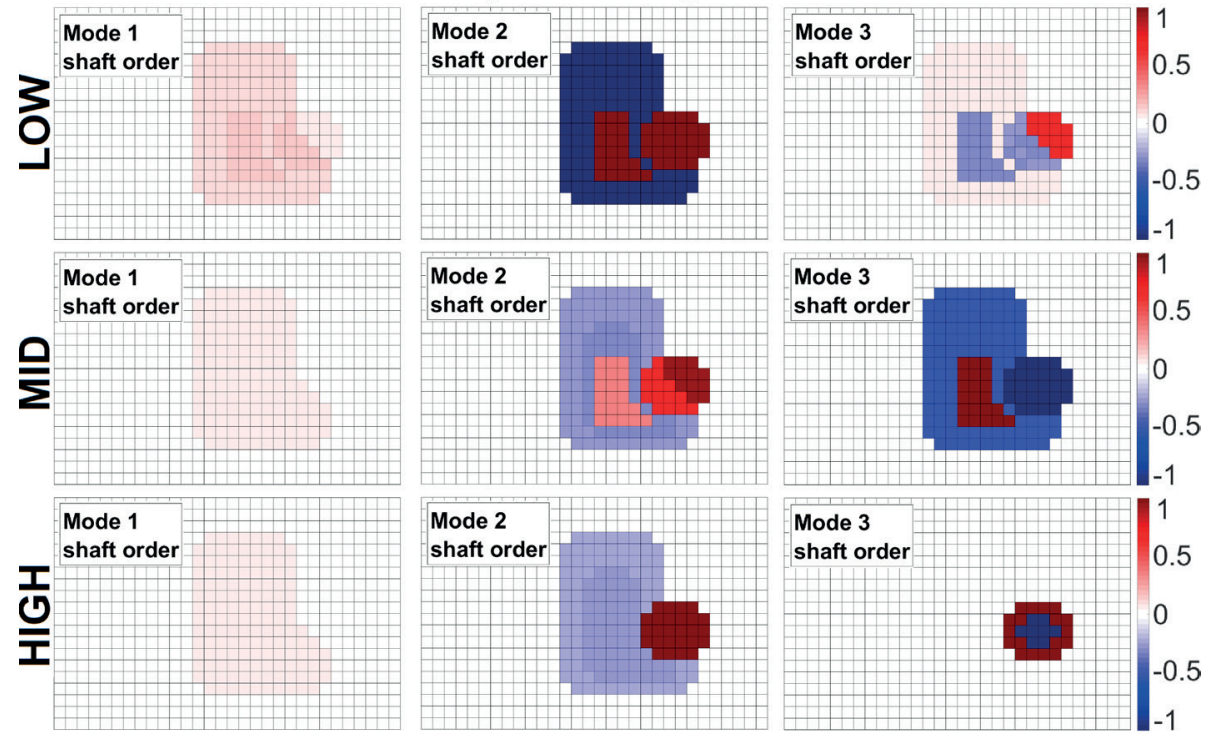

Fig. 10 Modal noise patterns, shaft order CPOD subsets for the model test cases, $2 \mathrm{~dB}$ 


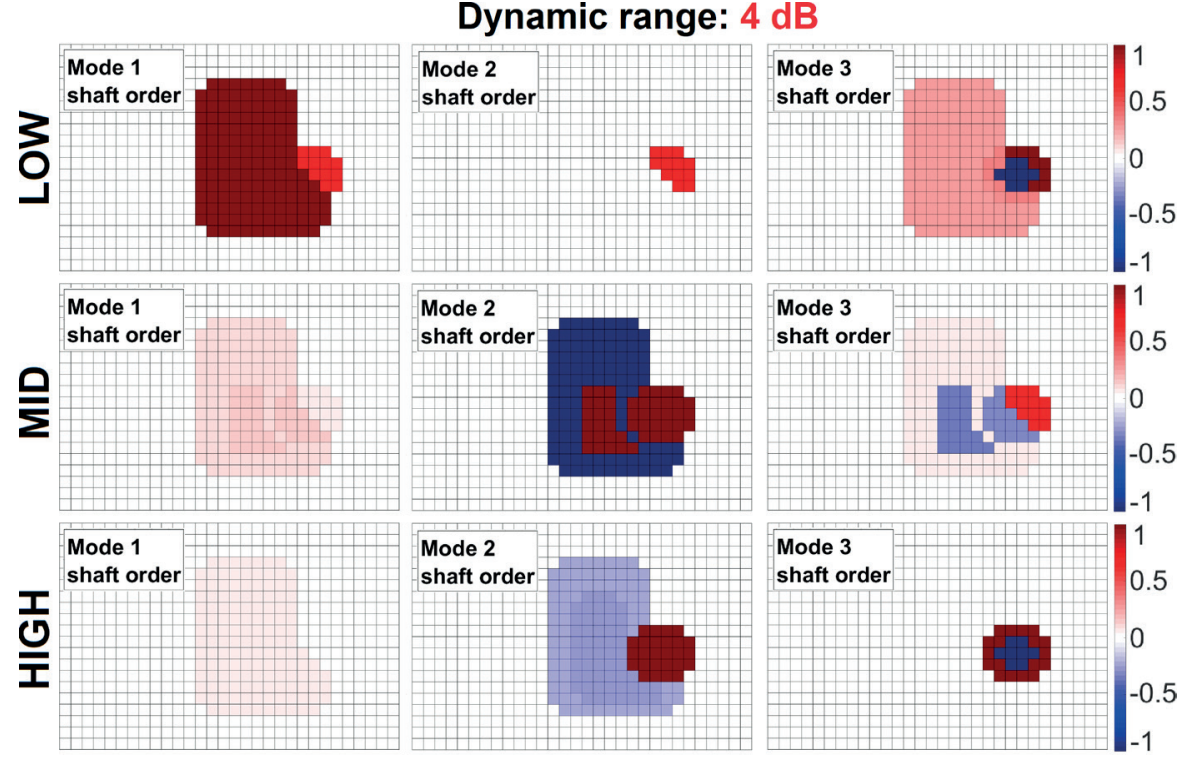

Fig. 11 Modal noise patterns, shaft order CPOD subsets for the model test cases, $4 \mathrm{~dB}$

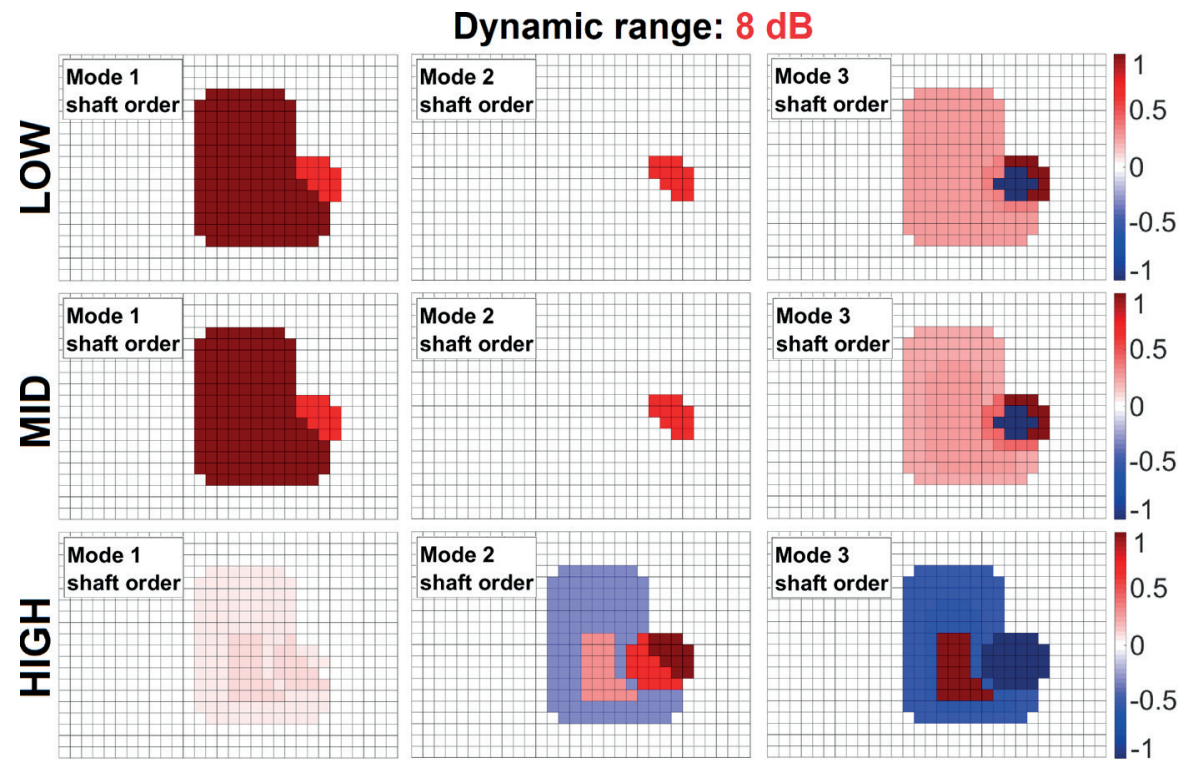

Fig. 12 Modal noise patterns, shaft order CPOD subsets for the model test cases, $8 \mathrm{~dB}$

$2 \mathrm{~dB}$ dynamic range as shown in Fig. 10. A second point that should be noticed is that Mode 1 is never dominated by the shaft order component for any of the test cases. This has been expected, as the shaft order component is expected to be among the less energetic contributors.

Based on the separation of the modal noise patterns on the CPOD maps, it can be stated that the characteristic locations of the dominant noise generation mechanisms can be identified for each of the principal components which have been determined by the PCA processing of the beamforming data set. However, due to the presence of the haystacking phenomenon on the input beamforming map (which is intentional for the model test case investigations), some of the energy is transferred between the modes, causing an overlap of the shaft order and broadband noise patterns regardless of the peak-to-broadband ratio. Nevertheless, the determination of the shapes pertaining to the investigated noise sources can still be carried out in the cases investigated to date, since values associated with the various corresponding modal noise source patterns tend to have alternate signs due to the variation of their weighting coefficients. Furthermore, as a direct result of the various peak-to-broadband ratios, the identification of the noise patterns of the shaft order tones is the best for the $H I G H$ model test case, for which the peak-to broadband ratio is the highest (see the bottom rows of Figs. 10 to 13). 


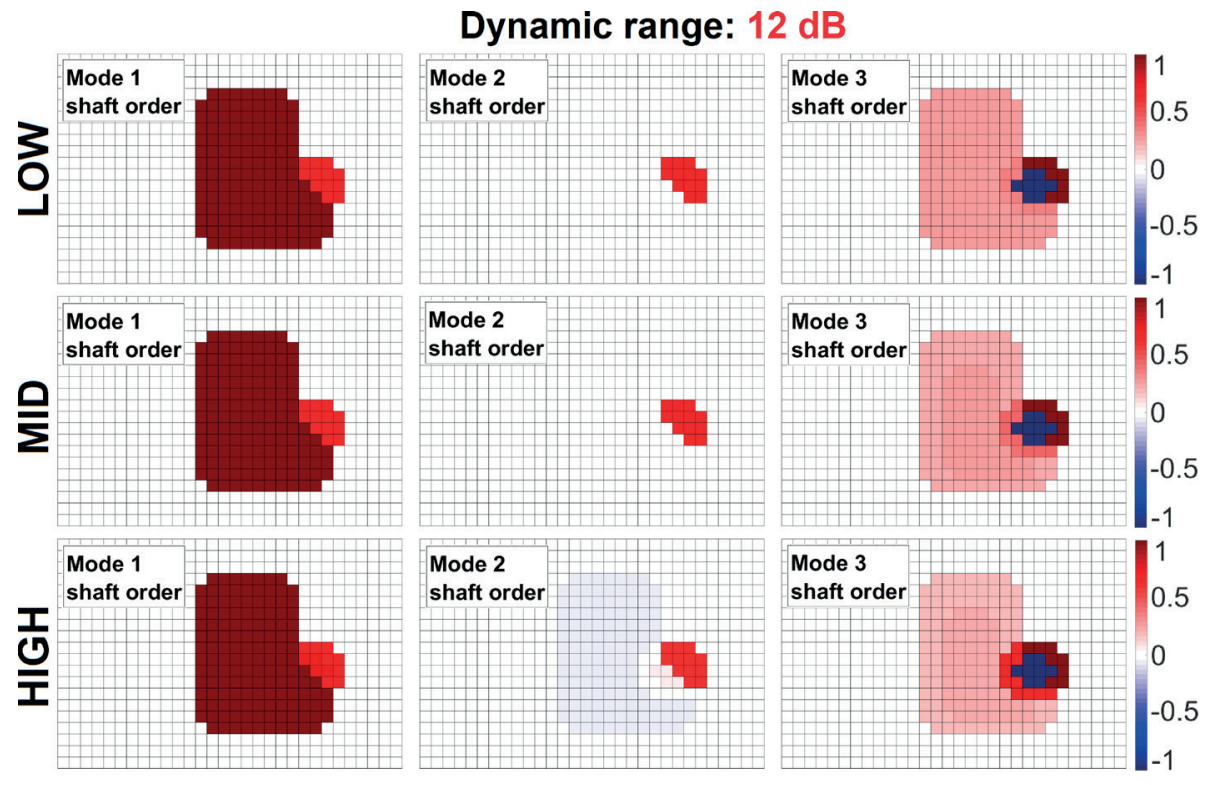

Fig. 13 Modal noise patterns, shaft order CPOD subsets for the model test cases, $12 \mathrm{~dB}$

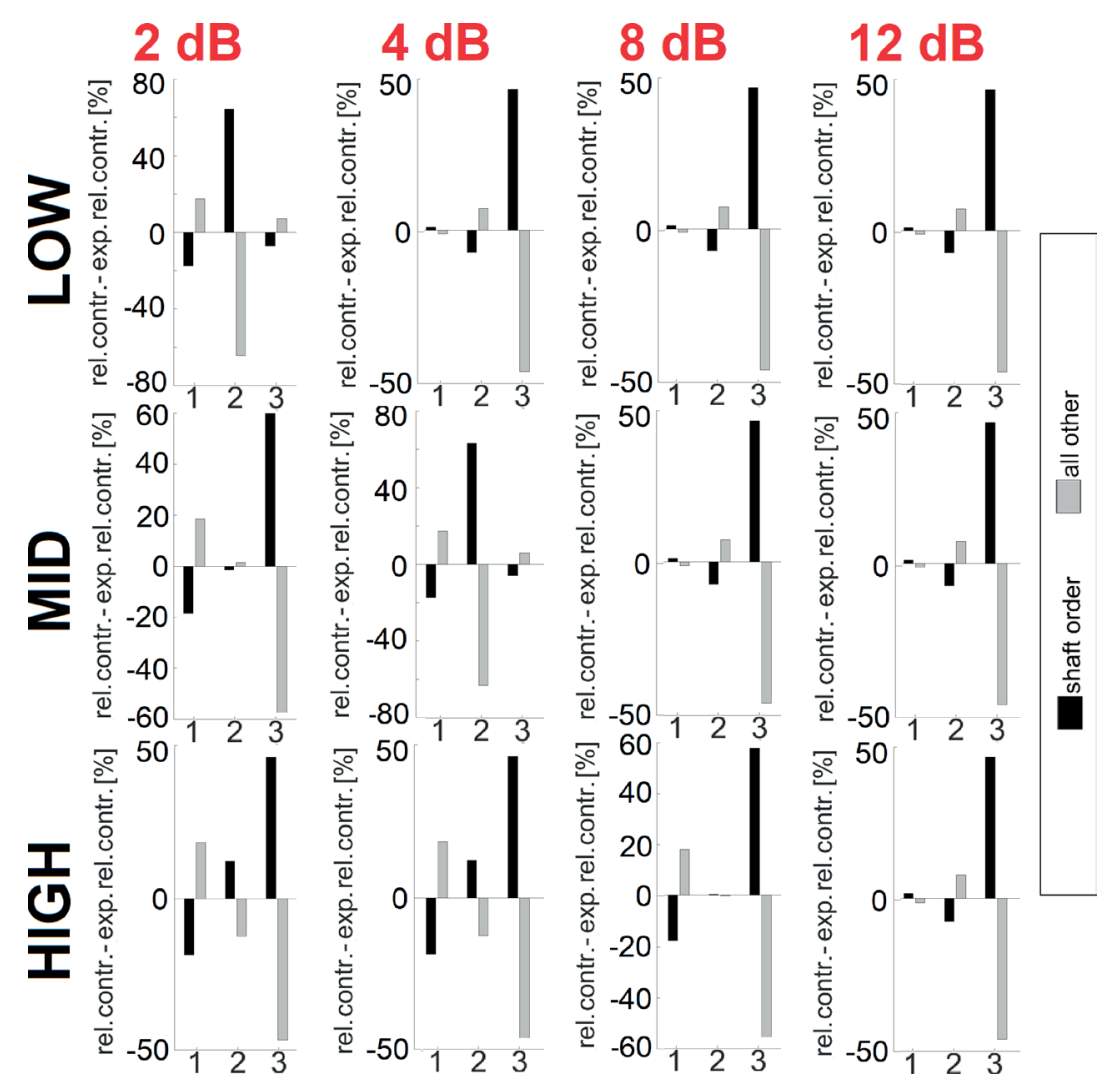

Fig. 14 Relative energy contributions, shaft order CPOD subsets for the model test cases

The effect of applying various dynamic ranges to the input beamforming maps has also been investigated. It has been found that the application of a small dynamic range can have a beneficial impact on the identification of the shaft order noise pattern. An improvement has been observed for the model test cases which contain input beamforming maps of lower peak-to-broadband ratios (e.g., for model test case $L O W$, as can be seen in the top row of Fig. 10). The reason for the beneficial effect is that the small dynamic range can mitigate the effect of the minimal rise of the tonal peak compared to the broadband. In comparison, the application of a dynamic range of high value can also be advantageous from another point of view, since less information is cut off artificially with the 
limitation of the dynamic range. Therefore all the noise source patterns are present for the PCA and CPOD methods to process (see Figs. 12, 13). On the other hand, this can make the input noise source maps very similar to each other if a large portion of the beamforming maps have levels which are near the upper bound of the dynamic range. This makes the separation of the noise sources more complicated. However, the opposite signs and different amplitudes of the modal noise patterns on the CPOD maps (see Fig. 13 for example) can still make the identification of the shaft order patterns possible. It also needs to be kept in mind that the application of a dynamic range of too large a value might be rather disruptive for a non-artificial test case, for which the presence of many other noise sources and sidelobes is expected. It has therefore been concluded that an optimal value of the applicable dynamic range can and should be found for any given test case, which is approximately equal to the value of the peak-to-broadband ratio. However, it has to be noted that a dynamic range smaller than this optimal value could also be suitable for test cases for which the average peak-to-broadband ratio at higher frequencies is considerably smaller than at lower frequencies. In addition, it is also advised to consider the applicable dynamic ranges with respect to the specific beamforming method used to create the input beamforming maps so that the dynamic range of the input beamforming maps does not exceed the value recommended for the applied beamforming method. The above conclusions resulting from the analysis of the model test cases are applied throughout the rest of the paper in the investigation of the CROR test case.

\subsection{Analysis of the CROR test case}

The PCA and CPOD analysis of the CROR data set has been conducted similarly to that of the model test cases. It has been found that the majority of the energy is contained in the first five modes. The modal noise patterns of the PCA and CPOD maps of these most energetic modes have been superimposed over the measurement setup for clarity. It has to be noted, that the maps have been enlarged and cropped in order to show only the close surroundings of the rotors which is relevant for the present test case study.

It is expected that the various energy contributions of the modes can be used to find the modes in which the energy contribution of the shaft order frequency bins are the most dominant, from which the corresponding noise patterns of the noise generation mechanism can be identified. The "rule of thumb" approximation presented above to determine the optimal dynamic range value of the input beamforming maps has been tested and further improved. As the peaks on the spectrum of the CROR test case do not have the same heights, it has to be investigated whether the peak-to-broadband ratios of the larger or the smaller peaks have to be used for the approximation of the optimal dynamic range. For the current CROR test case, the average peak-to-broadband value of the larger peaks is around $12 \mathrm{~dB}$, while the average peak-to-broadband value of the smaller peaks is around $4 \mathrm{~dB}$. Therefore, the data set has been investigated using various dynamic ranges from 2 to $12 \mathrm{~dB}$, in order to see which is most suitable for the identification of the shaft order pattern.

The complexity of the CROR test cases is considerably greater than that of the model test cases. Thus, it is also expected that the resulting modal noise patterns will not be as clean due to spatial interference and the potential overlaps of the various noise source patterns. Therefore, the corresponding modal noise patterns are expected to resemble the shapes and locations of the concentrated noise patterns associated with the investigated shaft order tones. In addition, it is also anticipated that the noise patterns show some characteristics that come from their overlapping with the BPF and broadband noise sources. The reason behind this is that rotating coherent BPF noise sources appear in every $12^{\text {th }}$ harmonic $\left(\mathrm{BPF}_{\mathrm{F}}\right)$ and $10^{\text {th }}$ harmonic $\left(\mathrm{BPF}_{\mathrm{A}}\right)$ of the fundamental shaft order frequency and the broadband noise sources appear in each and every frequency bin.

The relative energy contributions pertaining to the CPOD analysis of the beamforming data of the CROR test case with an applied dynamic range of $4 \mathrm{~dB}$ can be seen in Fig. 15. The PCA as well as the CPOD maps are shown in Fig. 16. Based on the examination of the relative energy contributions, it is found that Mode 5 is dominated by the frequency bins of shaft order tones. Even so, due to the presence of multiple noise generation mechanisms in each
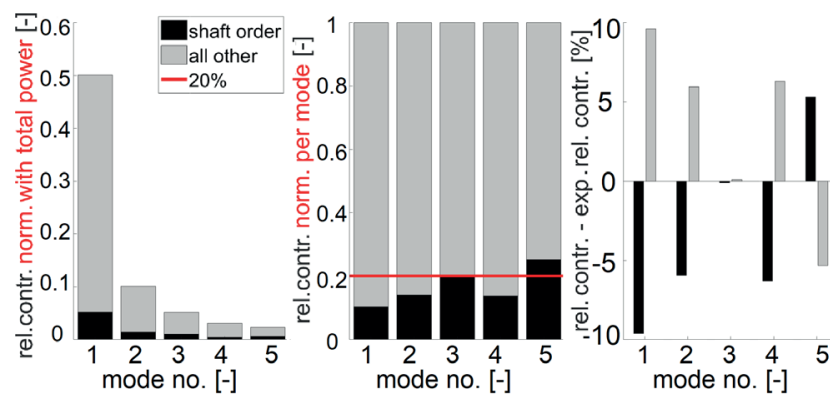

Fig. 15 Relative energy contributions of the first five modes, CROR test case, dynamic range: $4 \mathrm{~dB}$ 


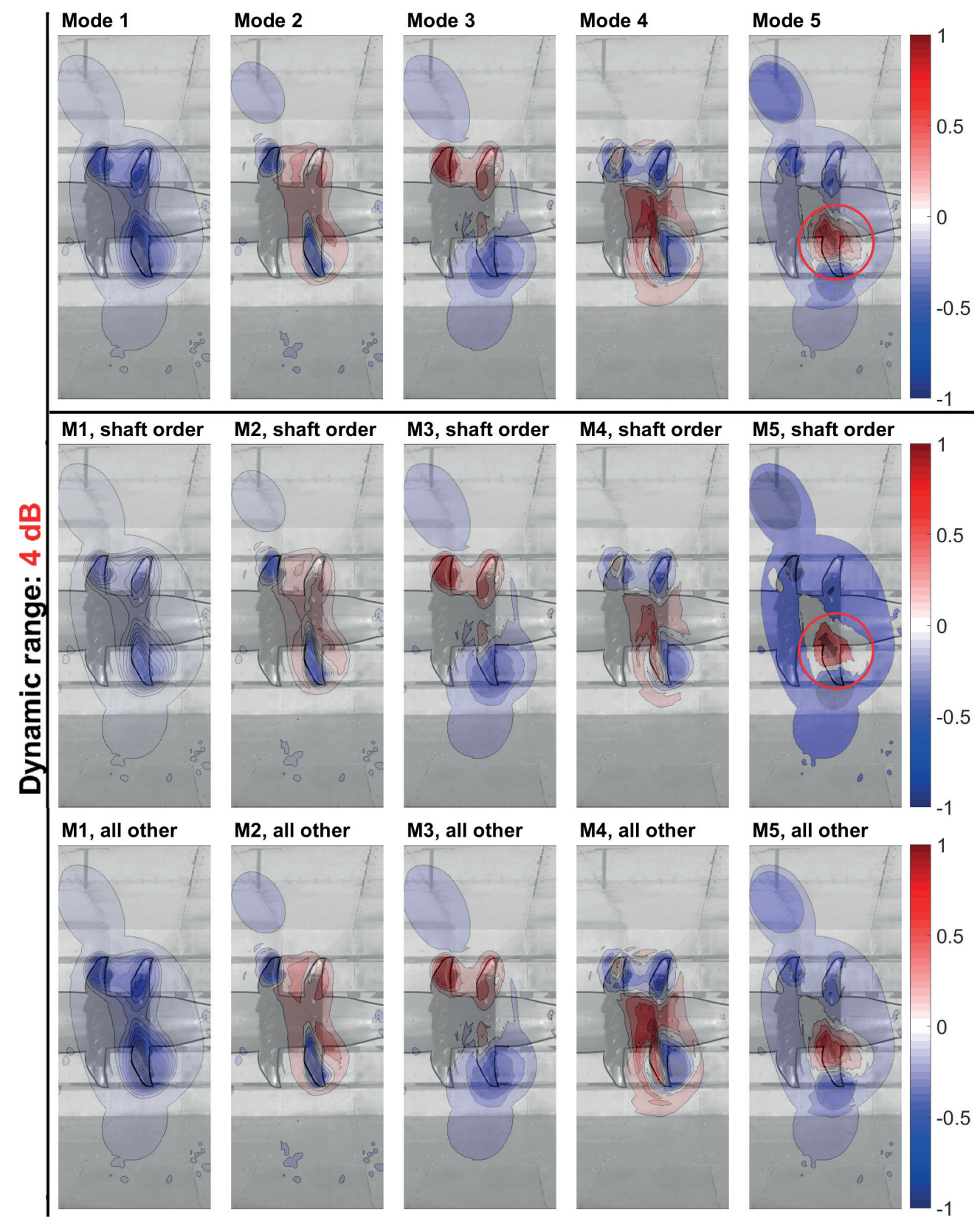

Fig. 16 Modal noise patterns of the first five modes, CROR test case, $4 \mathrm{~dB}$

of the frequency bins, the relative dominance of the shaft order tones is not entirely evident. Nonetheless, since values related to the various corresponding modal noise patterns have different signs and strengths, the localization of the shaft order tones (marked with a red circle on Fig. 16) is still possible. For Mode 5, the distinctive modal noise patterns of the investigated shaft order tones are characterized by positive values (different shades of red), in contrast to that of all the other noise source categories, which have negative values (blue). The conclusion of the CPOD analysis, namely that the shaft order noise pattern can be identified from the positive values in Mode 5, can be verified using the data presented in Subsection 4.2 in Fig. 2.
Furthermore, additional investigations have been carried out on the CROR data set, to ensure the accuracy of the PCA and CPOD based identification method. The effect of using input data sets comprised of different numbers of input beamforming maps on the CPOD identification process has been tested. It has been mentioned in Subsection 4.2 , that the currently investigated frequency range of the CROR test case starts at the $1^{\text {st }}$ BPFA and ends at the $10^{\text {th }}$. The BPFA has been divided into 50 bins of equal bandwidths, resulting in 451 frequency bins, i.e., input beamforming maps. For this bin independence study, various values for the upper limit of the investigated frequency range have been set. Therefore, the investigated frequency 
range starts at the $1^{\text {st }}$ BPFA in each case, but ends at the $5^{\text {th }}$, $6^{\text {th }}, 7^{\text {th }}, 8^{\text {th }}$ and $9^{\text {th }}$ BPFA, resulting in input beamforming map numbers of 201, 251, 301, 351 and 401 respectively. As such, by using fewer frequency bins, the size of the covariance matrix $\boldsymbol{R}$ (Eq. (2)) and the corresponding eigenvalue problem (Eq. (3)) changes. The influence of the number of frequency bins used in the investigation on the results is shown here by comparing the dominant modes of the shaft order tone. For each test case, the first CPOD modes in which the investigated noise pattern is dominant (thus the one having a relative energy contribution that rises above the expected contribution) have been examined. In Fig. 17 the CPOD maps corresponding to the shaft order $S$ ) subsets of these modes are shown for the test cases examined during the bin independency study. The figure is supplemented with the original test case presented in Fig. 16, for which a frequency range of $1^{\text {st }}$ to the $10^{\text {th }}$ BPFA has been applied (see the far right-hand side of Fig. 17). It can be seen that the noise patterns, which have been identified as shaft order noise patterns by the combined method, are almost identical to that of the original test case for the cases for which the upper limit of the investigated frequency range have been set to the $7^{\text {th }}, 8^{\text {th }}$ and $9^{\text {th }}$ BPFA. Furthermore, the cases for which an upper limit of the 5th and 6th BPFA has been set still reveal very similar noise patterns to that of the original CROR test case, but its shape and location are somewhat shifted. Therefore, the bin independence study has shown that the combined method presented herein is capable of identifying the investigated noise pattern even when a considerably smaller input data set is used.

The effect on the CPOD maps of applying various dynamic ranges has also been examined. The effect on the CPOD maps of applying various dynamic ranges is shown in Fig. 18. It has been determined that the noise source pattern associated with the shaft order frequency bins is dominant in Mode 5 for the data sets of beamforming maps with the applied dynamic ranges of 2, 4 and 12, while for the case when a dynamic range of $8 \mathrm{~dB}$ has been used, Mode 4 is dominant with respect to the shaft order subset. It has been found, that although applying a $2 \mathrm{~dB}$ dynamic range the effect of haystacking as well as the spatial interference of non-shaft-order sources are lowered, valuable information is lost in the process. Therefore, while the shaft order pattern is present on the CPOD map, it cannot be clearly identified. When a dynamic range of $4 \mathrm{~dB}$ has been applied, it can be stated that the percentage difference of the relative energy contribution is the highest among the shaft order dominated modes of the dynamic range investigations. When looking at the CPOD map associated with this shaft order dominant mode (i.e., Mode 5), it can be seen that the shaft order pattern can be clearly identified (marked with a red circle). Therefore, it can be stated, that an applied dynamic range of $4 \mathrm{~dB}$ is suitable for the current $\mathrm{CROR}$ test case. In contrast, the results pertaining to the larger dynamic ranges of 8 and $12 \mathrm{~dB}$ have been found to be difficult to interpret. The shaft order noise pattern can partly be seen, as the applied dynamic range is relatively large. However, sidelobes and many additional noise generation mechanisms are also present, making it harder (or even impossible) to identify the noise source pattern of the shaft order. Consequently, as the dynamic range of $4 \mathrm{~dB}$ is equal to the average peak-to-broadband value of the smaller tonal peaks, the "rule of thumb" approximation presented in Subsection 6.1 has been extended with the following guideline. In order to best identify the noise patterns using the CPOD method, the applied dynamic range is to be set to equal the value of the average peak-to-broadband ratio of the smaller tonal peaks.
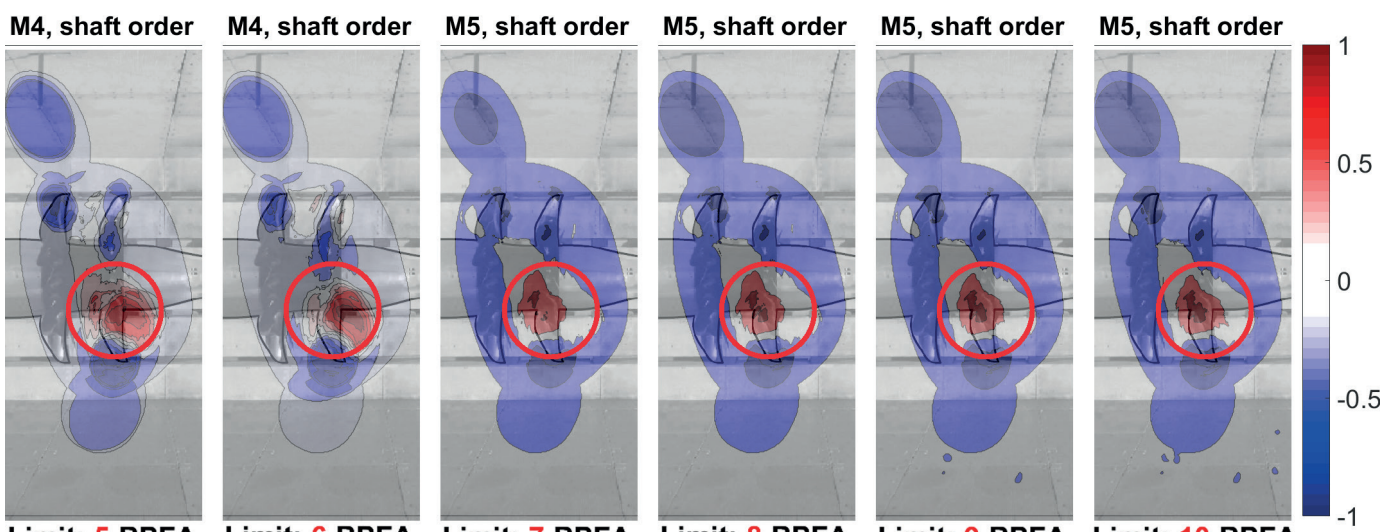

Fig. 17 Modal noise patterns of the most dominant shaft order modes for different input map numbers, $4 \mathrm{~dB}$ 

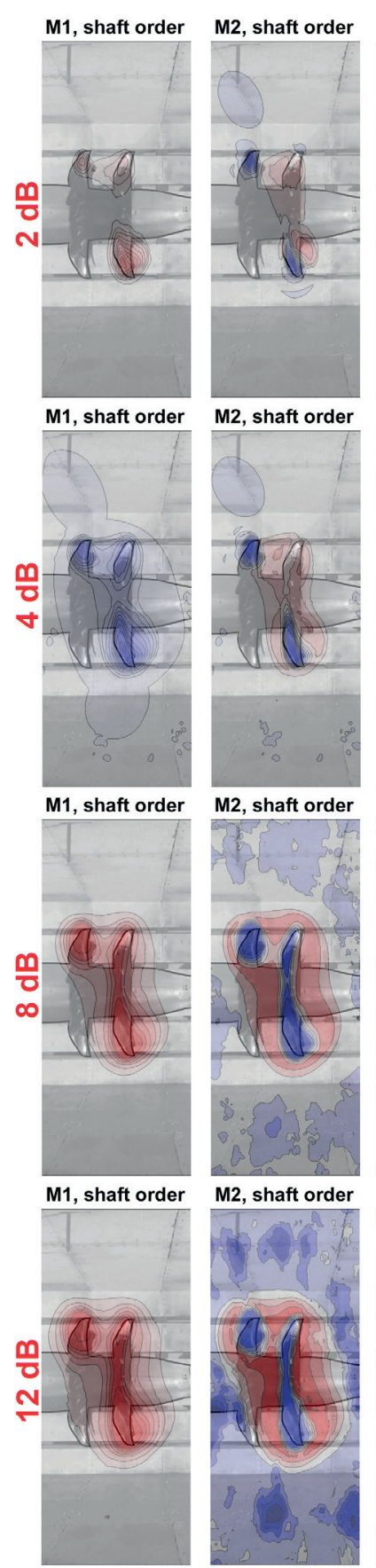
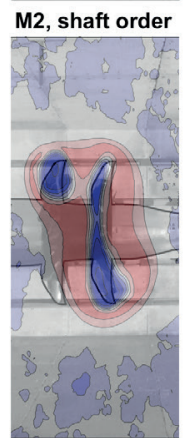

M2, shaft order

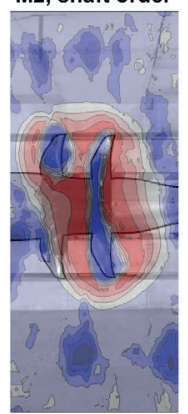

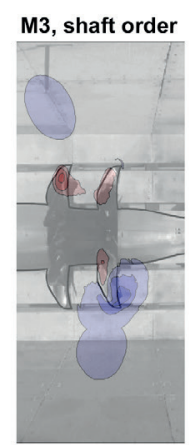

M3, shaft order

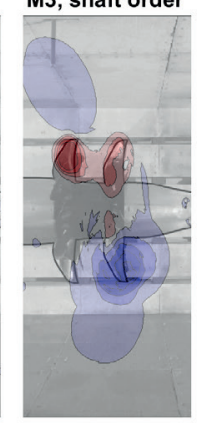

M3, shaft order

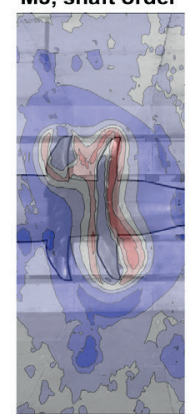

M3, shaft order

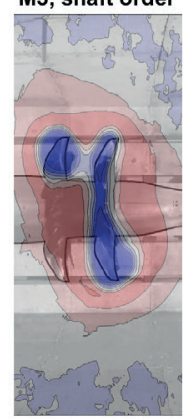

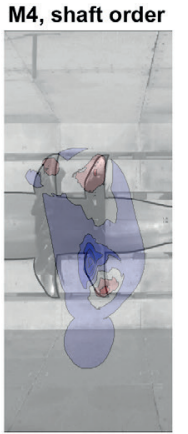

M4, shaft order

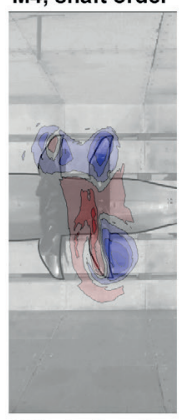

M4, shaft order

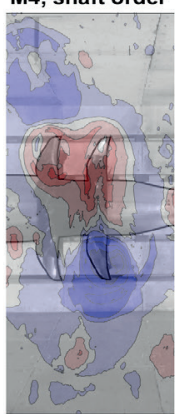

M4, shaft order

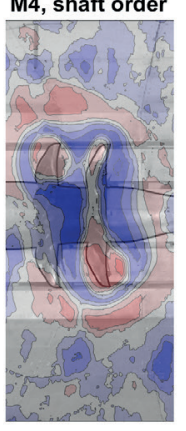

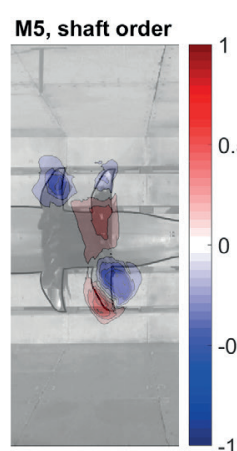

M5, shaft order

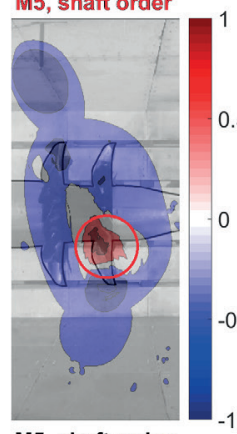

M5, shaft order
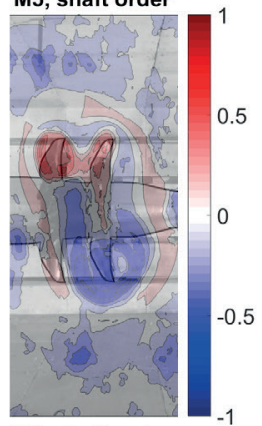

M5, shaft order

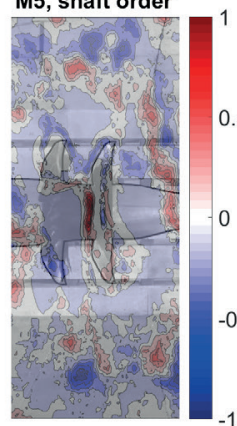

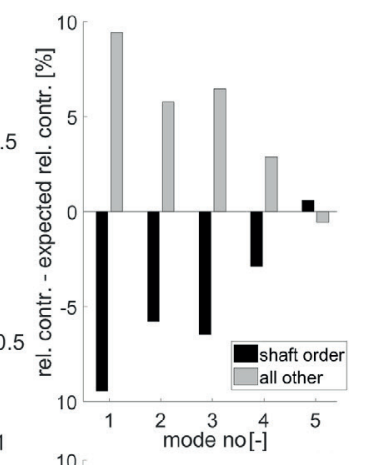

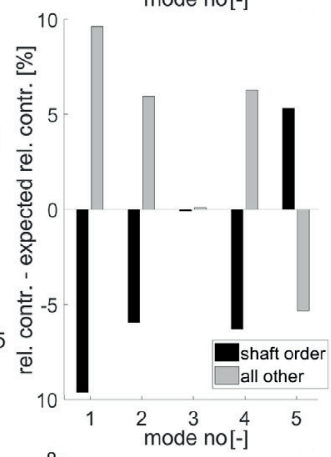

8
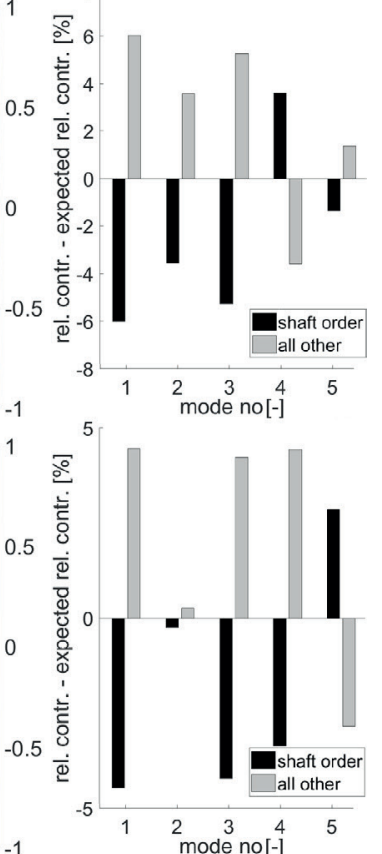

Fig. 18 CPOD maps of the first five modes (shaft order subset)

\section{Conclusion}

The combined implementation of processing beamforming results using principal component analysis and common-base proper orthogonal decomposition has been studied by investigating counter-rotating open rotor noise. The main steps can be summarized as follows. The method starts with phased array measurements, then the beamforming maps are created using beamforming in the frequency domain. Next, the optimal plot dynamic range of the beamforming maps has to be determined in order to create suitable inputs for the CPOD based identification method. In order to identify the characteristic noise source patterns of a chosen noise source category, an initial PCA analysis is performed. Subsets associated with the investigated noise source categories are determined. CPOD is then used to analyze the relative energy contributions of the subsets and visualize their impacts in order to pinpoint modes in which the noise source patterns of the investigated noise sources are to be found and identified. 
After conducting an initial analysis on artificially generated model test cases, the investigation of the CROR data set has been performed. It has been concluded that CPOD analysis is an effective method for giving the researchers guidance in identifying and localizing noise sources with various energy contributions without a time consuming sorting process. The method applied herein is able to extract even weak patterns that repeat throughout the domain of the set of beamforming maps under investigation. The analysis yields results that are easy to comprehend without special prior knowledge, which is an important advancement compared to the formerly used manual sorting method. It has also been found that the increase of the peak-to-broadband ratio significantly improved the identification and separation of the noise source patterns. The data sets have also been examined with multiple plot dynamic range settings with regard to the input beamforming maps. It has been found that while a larger dynamic range can be beneficial as it contains more information for the PCA and CPOD algorithms, it should still be chosen carefully, since the limited peak-to-broadband and mainlobe-to-sidelobe ratios of the beamforming results can have a negative effect on the quality of the separation and detection of the various spatial noise patterns of the noise sources involved in the investigation. It has

\section{References}

[1] Peake, N., Parry, A. B. "Modern Challenges Facing Turbomachinery Aeroacoustics", Annual Review of Fluid Mechanics, 44(1), pp. 227-248, 2012.

https://doi.org/10.1146/annurev-fluid-120710-101231

[2] Dickson, N. "ICAO Noise Standards", [pdf] presented at ICAO Symposium on Aviation and Climate Change, Montréal, QC, Canada, May, 14-16, 2013, Available at: https://www.icao.int/ Meetings/Green/Documents/day\%201pdf/session\%202/2-Dickson. pdf [Accessed: 29 September 2020]

[3] Dougherty, R. P. "Beamforming In Acoustic Testing", In: Mueller, T. J. (ed.) Aeroacoustic Measurements, Springer, Berlin, 2002, pp. 62-97.

https://doi.org/10.1007/978-3-662-05058-3_2

[4] Horváth, C. "Beamforming Investigation of Dominant CounterRotating Open Rotor Tonal and Broadband Noise Sources", AIAA Journal, 53(6), pp. 1602-1611, 2015.

https://doi.org/10.2514/1.J053465

[5] Fenyvesi, B., Tokaji, K., Horváth C. "Investigation of a Pylons Effect on the Character of Counter-Rotating Open Rotor Noise Using Beamforming Technology", Acta Acustica united with Acustica, 105(1), pp. 56-65, 2019.

https://doi.org/10.3813/AAA.919287

[6] Horváth C., Envia, E., Podboy, G. G. "Limitations of Phased Array Beamforming in Open Rotor Noise Source Imaging", AIAA Journal, 52(8), pp. 1810-1817, 2014. https://doi.org/10.2514/1.J052952 been determined that an optimal value of plot dynamic range can be found for any investigated test case, which is approximately equal to the value of the peak-to-broadband ratio. In conclusion, it can be stated that the combined method presented herein can be used for the analysis of various kinds of turbomachinery noise sources.

\section{Acknowledgement}

The testing of the CROR was funded by the Environmentally Responsible Aviation Project of the NASA Integrated Systems Research Program and the Fixed Wing Project of the NASA Fundamental Aeronautics Program. The research presented in this paper has been supported by the Hungarian National Research, Development and Innovation Centre under contract No. K 129023, and the János Bolyai Research Scholarship of the Hungarian Academy of Sciences, by the ÚNKP-21-5 New National Excellence Program of the Ministry of Human Capacities, and by the NRDI Funds (TKP2020 IES, Grant No. BMEIE-MISC) and (TKP2020 NC, Grant No. BME-NCS) based on the charter of bolster issued by the NRDI Office under the auspices of the Ministry for Innovation and Technology. The authors acknowledge financial support from the DAAD Eastern Partnership (2019-2021).

[7] Pearson, K. "LIII. On lines and planes of closest fit to systems of points in space", The London, Edinburgh, and Dublin Philosophical Magazine and Journal of Science, 2(11), pp. 559-572, 1901. https://doi.org/10.1080/14786440109462720

[8] Hotelling, H. "Analysis of a complex of statistical variables into principal components", Journal of Educational Psychology, 24(6), pp. 417-441, 1933. https://doi.org/10.1037/h0071325

[9] Kriegseis, J., Dehler, T., Gnirß, M., Tropea, C. "Common-base proper orthogonal decomposition as a means of quantitative data comparison", Measurement Science and Technology, 21(8), Article number: 085403, 2010.

https://doi.org/10.1088/0957-0233/21/8/085403

[10] Fenyvesi, B., Simon, E., Kriegseis, J., Horváth, C. "Investigation of Turbomachinery Noise Sources Using Beamforming Technology and Proper Orthogonal Decomposition Methods", [pdf] In: Conference on Modelling Fluid Flow (CMFF'18), The 17th International Conference on Fluid Flow Technologies, Budapest, Hungary, 2018, pp. 1-8, Available at: http://real.mtak.hu/85324/1/ CMFF18_032_u.pdf [Accessed: 29 September 2020]

[11] Woodward, R. P. "Noise of a model high speed counterrotation propeller at simulated takeoff/approach conditions (F7/A7)", In: 11th Aeroacoustics Conference, Sunnyvale, CA, USA, Technical Memorandum 100206, AIAA-87-2657 1987. https://doi.org/10.2514/6.1987-2657 
[12] Hanson, D. B. "Noise of counter-rotation propellers", Journal of Aircraft, 22(7), pp. 609-617, 1985.

https://doi.org/10.2514/3.45173

[13] Fenyvesi, B., Kriegseis, J., Horváth, C. "Application of a Combined Method for the Investigation of Turbomachinery Noise Sources: Beamforming and Proper Orthogonal Decomposition", In: 25th AIAA/CEAS Aeroacoustics Conference, Delft, The Netherlands, 2019, pp. 1-17.

https://doi.org/10.2514/6.2019-2637

[14] Fenyvesi, B., Kriegseis, J., Horváth, C. "Investigation of Rotating Coherent BPF Noise Sources via the Application of Beamforming and Proper Orthogonal Decomposition", In: INTER-NOISE and NOISE-CON Congress and Conference Proceedings, Madrid, Spain, 2019, pp. 3127-3138, [online] Available at: https://www.ingentaconnect.com/content/ince/incecp/2019/00000259/00000006/ art00013 [Accessed: 29 September 2020]

[15] Karhunen, K. "Zur Spektraltheorie Stochasticher Prozesse" (On the spectral theory of stochastic processes), Suomalainen Tiedeakatemia, Annales Academiae Scientiarum Fennicae, Series A1, Helsinki, Finnland, 1946.

[16] Loève, M. "Elementary Probability Theory", In: Loève, M. (ed.) Probability Theory I, Springer, New York, NY, USA, 1977, pp. 1-52. https://doi.org/10.1007/978-1-4684-9464-8_1

[17] Lumley, J. L. "The Structure of Inhomogeneous Turbulent Flows", In: Yaglom, A. M., Tatarsky, V. I. (eds.) Atmospheric Turbulence and Radio Wave Propagation, Nauka, Moscow, SU, 1967, pp. $166-178$.

[18] Mueller Stein, S. A., Loccisano, A. E., Firestine, S. M., Evanseck, J. D. "Chapter 13 Principal Components Analysis: A Review of its Application on Molecular Dynamics Data", In: Spellmeyer, D. C. (ed.) Annual Reports in Computational Chemistry, Elsevier, 2006, pp. 233-261. https://doi.org/10.1016/S1574-1400(06)02013-5

[19] Preisendorfer, R. W., Mobley, C. D. "Principal component analysis in meteorology and oceanography", Developments in atmospheric science, 17, Article number: XVIII-425, 1988, [online] Available at: http://pascal-francis.inist.fr/vibad/index.php?action=getRecordDetail\&idt=7118566 [Accessed: 29 September 2020]
[20] Meyer, K. E., Pedersen, J. M., Özcan, O. "A turbulent jet in crossflow analysed with proper orthogonal decomposition", Journal of Fluid Mechanics, 583, pp. 199-227, 2007. https://doi.org/10.1017/S0022112007006143

[21] Berkooz, G., Holmes, P., Lumley, J. L. "The Proper Orthogonal Decomposition in the Analysis of Turbulent Flows", Annual Review of Fluid Mechanics, 25(1), pp. 539-575, 1993. https://doi.org/10.1146/annurev.fl.25.010193.002543

[22] Liang, S., Chen, W., Liem, R. P., Huang, X. "Experimental Analysis of Cooling Fan Noise by Wavelet-Based Beamforming and Proper Orthogonal Decomposition", IEEE Access, 8, pp. 121197-121203, 2020.

https://doi.org/10.1109/ACCESS.2020.3006483

[23] Van Zante, D. E., Gazzaniga, J. A., Elliott, D. M., Woodward, R. P. "An open rotor test case: F31/A31 historical baseline blade set", In: 20th International Symposium on Airbreathing Engines, Gothenburg, Sweden, 2011, pp. 2011-1310.

[24] OptiNav Inc. "Array 48: Microphone Phased Array System", [pdf] Available at: https://www.optinav.info/Array48.pdf [Accessed: 02 May 2021]

[25] Sharland, I. J. "Sources of noise in axial flow fans", Journal of Sound and Vibration, 1(3), pp. 302-322, 1964. https://doi.org/10.1016/0022-460X(64)90068-9

[26] Blandeau, V. "Aerodynamic broadband noise from contra-rotating open rotors", PhD thesis, University of Southampton, 2011. [online] Available at: https://eprints.soton.ac.uk/179853/ [Accessed: 29 September 2020]

[27] Magliozzi, B., Hanson, D. B., Amiet, R. K. "Propeller and Propfan Noise", In: Hubbard, H. H. (ed.) Aeroacoustics of Flight Vehicles: Theory and Practice. Volume 1: Noise Sources, Hampton, VA, US, 1991, pp. 1-61. [online] Available at: https://ntrs.nasa.gov/citations/19920001380 [Accessed: 29 September 2020]

[28] Kingan, M. J. "Open rotor broadband interaction noise", Journal of Sound and Vibration, 332(17), pp. 3956-3970, 2013. https://doi.org/10.1016/j.jsv.2013.03.014 